\title{
Synthesis and molecular structure of novel 2-(alkylthio)-4-chloro- $N$-(4,5-dihydro-5-oxo-1H-1,2,4-triazol-3-yl)-5-methylbenzene- sulfonamides with potential anticancer activity
}

\author{
Jarosław Sławiński • Beata Żołnowska • \\ Czesława Orlewska $\cdot$ Jarosław Chojnacki
}

Received: 1 March 2012/ Accepted: 22 August 2012/Published online: 25 September 2012

(C) The Author(s) 2012. This article is published with open access at Springerlink.com

\begin{abstract}
A series of novel 4-chloro- $N$-(4,5-dihydro-5-oxo1- $\mathrm{R}^{2}-1 H-1,2,4-$ triazol-3-yl)-5-methyl-2-( $\mathrm{R}^{1}$-methylthio)benzenesulfonamide derivatives have been synthesized as potential anticancer agents. The in vitro antitumor activity of some compounds was evaluated in the US National Cancer Institute (NCI) against the NCI-60 cell line panel. The most prominent compound showed remarkable activity against 13 human tumor cell lines representing lung, colon, CNS, melanoma, ovarian, renal, prostate, and breast at low micromolar $\mathrm{GI}_{50}$ level in the range of $1.9-3.0 \mu \mathrm{M}$.
\end{abstract}

Keywords Sulfonamide - Anticancer · Isocyanate · Cyclization · Triazolone

\section{Introduction}

The aryl- and heteroarylsulfonamides are widely described compounds revealing a broad spectrum of applications in biological and pharmacological areas [1]. For many years, 2-mercaptobenzenesulfonamide derivatives (MBSAs) have been of interest because of the various biological properties including antitumor [2-10], antimicrobial [11, 12], and antiviral activities [13, 14], and inhibition of carbonic anhydrase [15-17].

Electronic supplementary material The online version of this article (doi:10.1007/s00706-012-0849-7) contains supplementary material, which is available to authorized users.

J. Sławiński $(\square) \cdot$ B. Żołnowska · C. Orlewska

Department of Organic Chemistry, Medical University of

Gdańsk, Gen. J. Hallera Str. 107, 80416 Gdańsk, Poland

e-mail: jaroslaw@gumed.edu.pl

J. Chojnacki

Department of Chemistry, Gdańsk University of Technology,

Narutowicza 11/12, 80233 Gdańsk, Poland
It has been known that aryl/heteroarylsulfonamides may act as antitumor agents through a variety of mechanisms such as cell cycle perturbation in the G1 phase, disruption of microtubules, angiogenesis inhibition, and functional suppression of the transcriptional activator NF-Y. The most prominent mechanism was the inhibition of carbonic anhydrase isozymes [18-22]. Recently, a host of structurally novel arylsulfonamide derivatives have been reported to show substantial anticancer activities in vitro and/or in vivo [23-26]. We have reported the synthesis and anticancer activity of 2-mercaptobenzenesulfonamides and subsequently extended our study to analogues with various heterocyclic ring systems attached to the benzenesulfonamide scaffold [4-6, 8, 10, 15] (Fig. 1 structure A [4-6, 8, 15], B [8], C [10]).

In this article we investigated new sulfonamide derivatives containing a triazolone ring in their structure. Triazolones are described in the literature as biologically active compounds, including anti-inflammatories [27], $\mathrm{Nk}_{\mathrm{i}}$ antagonists [28], inhibitors of tumor necrosis factor- $\alpha$-converting enzyme (TACE) [29], checkpoint kinase-1 inhibitor [30], anti-tumor agents [31-34], and molecular chaperone Hsp90 inhibitor, which is currently in clinical trials for a number of human cancers [35]. Taking into account the interesting properties of triazolones, we have synthesized novel compounds of general structure D (Fig. 1).

\section{Results and discussion}

\section{Chemistry}

The main goal of this study was to synthesize and investigate the anticancer activity of the new 2-(alkylthio)benzenesulfonamides containing diverse substituted 1,2,4-triazol-5-one moieties. Thus, we propose a synthetic route leading to the target 
Fig. 1 General structures of 2-mercaptobenzenesulfonamides $\mathbf{A}, \mathbf{B}, \mathbf{C}$, and $\mathbf{D}$<smiles>[R]CSc1cc(Cl)c([R])cc1S(=O)(=O)NCl</smiles>

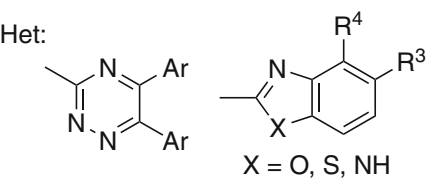<smiles>[Z]C(C)=O</smiles>
$Z=$ aromatic or
heteroaromatic ring<smiles>[Z]C=NNC(C)=N</smiles>

A<smiles>[R]NC(=NS(=O)(=O)c1cc([R])c(Cl)cc1S[R])N/N=C/c1ccc([N+](=O)[O-])o1</smiles><smiles>[R7]c1cc(S(=O)(=O)/N=C(\N)n2nc([R])c([R])c2[R])c(SCc2ccccc2)cc1Cl</smiles>

C<smiles>[R]CSc1cc(Cl)c(C)cc1S(=O)(=O)Nc1nn(P)c(=O)[nH]1</smiles>

\section{Scheme 1}

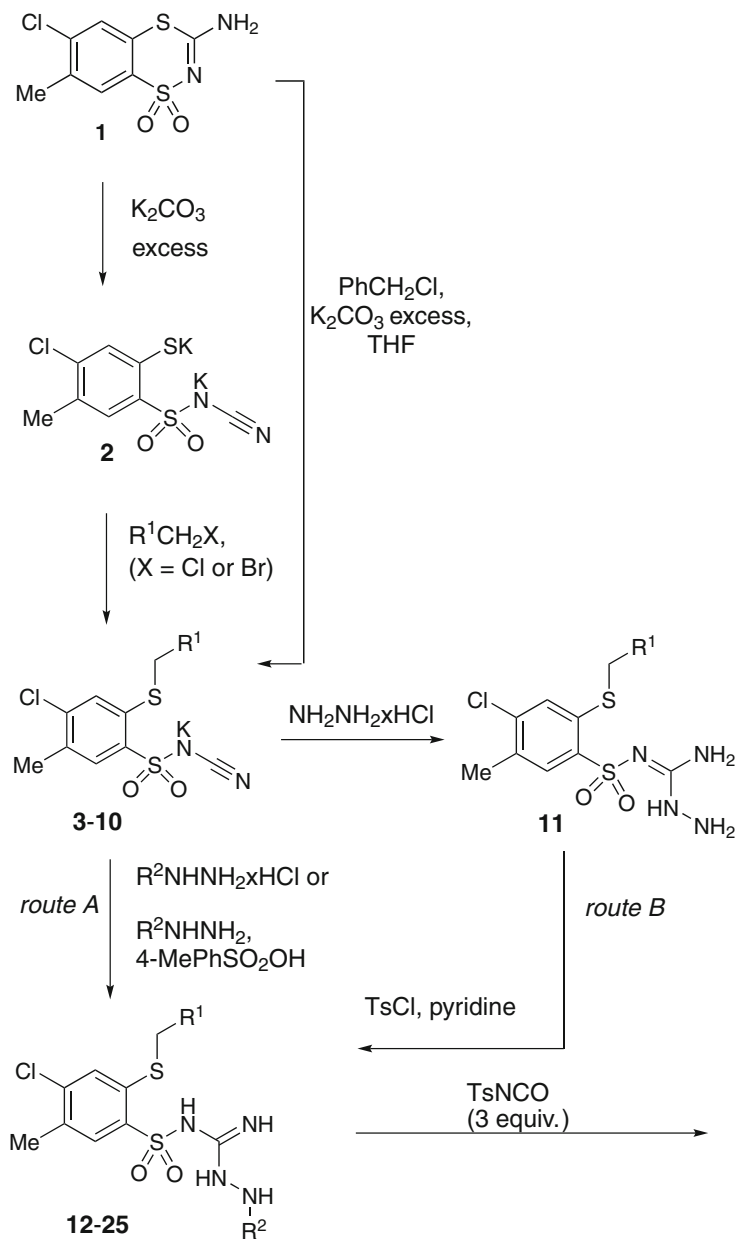

\begin{tabular}{|c|c|c|}
\hline Compounds & $\mathrm{R}^{1}$ & $R^{2}$ \\
\hline $3,11,26$ & $\mathrm{Ph}$ & $\mathrm{H}$ \\
\hline $3,12,27$ & $\mathrm{Ph}$ & $\mathrm{Me}$ \\
\hline $3,13,28$ & $\mathrm{Ph}$ & $\mathrm{Ph}$ \\
\hline $3,14,29$ & $\mathrm{Ph}$ & $4-\mathrm{O}_{2} \mathrm{NPhSO}_{2}$ \\
\hline $3,15,30$ & $\mathrm{Ph}$ & $4-\mathrm{MePhSO}_{2}$ \\
\hline $4,16,31$ & COOEt & 4-MePh \\
\hline $5,17,32$ & & $\mathrm{Ph}$ \\
\hline $6,18,33$ & $3-\mathrm{CF}_{3} \mathrm{Ph}$ & $\mathrm{Ph}$ \\
\hline $6,19,34$ & 3- $\mathrm{CF}_{3} \mathrm{Ph}$ & 4-MePhSO ${ }_{2}$ \\
\hline $7,20,35$ & $4-\mathrm{CF}_{3} \mathrm{Ph}$ & $\mathrm{Ph}$ \\
\hline $8,21,36$ & 1-naphthyl & $\mathrm{Ph}$ \\
\hline $8,22,37$ & 1-naphthyl & 4-CIPh \\
\hline $8,23,38$ & hthyl & 4-MePhSO 2 \\
\hline $9,24,39$ & & $\mathrm{Ph}$ \\
\hline $10,25,40$ & & $\mathrm{Ph}$ \\
\hline $6-4$ & & \\
\hline
\end{tabular}

2-(alkylthio)-4-chloro- $N$-(4,5-dihydro-5-oxo-1-R ${ }^{2}-1 H-1,2,-$ 4-triazol-3-yl)-5-methylbenzenesulfonamides as shown in Scheme 1.

Starting 3-aminobenzodithiazine $\mathbf{1}$ could be readily converted to the corresponding dipotassium 2 and potassium salts 3 and 4, according to the reported procedure for preparation of $N$-(phenylsulfonyl)cyanamide potassium salts [36]. Novel potassium salts 5-10 were prepared by the reaction of $\mathbf{2}$ with the corresponding halomethyl electrophiles such as aryl/cycloalkyl/methyl chlorides in methanol or ethanol. 
Subsequent reaction of salts 3-10 with either hydrazine monohydrochloride, methylhydrazine, $p$-toluenesulfonyl hydrazide, or various 4-substituted phenylhydrazine hydrochlorides led to the formation of the desired 3-( $\mathrm{R}^{2}$-amino $)-1$ [4-chloro-5-methyl-2-( $\mathrm{R}^{1}$-methylthio)phenylsulfonyl]guanidine derivatives 11-25 as depicted in Scheme 1. It is pertinent to know, however, that aminoguanidine $\mathbf{1 5}$ was chosen for the synthesis in two different ways (route A and B in Scheme 1). This was supposed to explain some arising synthetic aspects: whether the usefulness of the potassium salt, i.e., $\mathbf{3}$ with tosyl hydrazide (route A), is higher than the reaction of aminoguanidine 11 with tosyl chloride ( $\mathrm{TsCl}$, route $\mathrm{B}$ ), and whether the reaction proceeds on the $\mathrm{N}$-terminal nitrogen atom of the sulfonylhydrazide moiety or on the second nitrogen atom neighboring the sulfonyl group. As it turned out, both methods products $\mathbf{1 5}$ were identical, with structures (IR, NMR) having a $N$ '-substituted sulfonylhydrazide fragment and obtained in almost equal yields.

Many methods are known for the synthesis of 1,2,4-triazol5-ones. Triazol-5-ones can be prepared for instance by the reaction of the corresponding nitriles via imidates with semicarbazide [37], from 4-substituted semicarbazides under alkaline conditions [32], by heating of $N^{1}, N^{4}$-substituted hydrazinecarboxamides in alkaline media [38], by cyclization of semicarbazide with an excess of phosgene [39], from the reaction of $\mathrm{N}$-acylureas with arylhydrazines [40], $\mathrm{N}$-acylurethanes with phenylhydrazines as an Einhorn-Brunner reaction extension, as well as from $C$-halobenzylidenephenylhydrazones via nitrilimines with phenyl isocyanates [41, 42].

In the present study we utilized a new method for the synthesis of 1,2,4-triazol-5-ones in the reaction of the corresponding aminoguanudines 11-25 with an excess of $p$-toluenesulfonyl isocyanate (TsNCO, Scheme 1). The isocyanates are well known as carbonyl precursors [43] and electrophilic agents whose reactions with hydrazines lead to intramolecular cyclization to five-membered heterocyclic rings [44] or reagents in cycloaddition reactions with various compounds having $\mathrm{C}=\mathrm{N}$ bonds [45].

Our experiments demonstrated that the proposed synthetic route was an efficient way to prepare the desired $N$-(4,5-dihydro-5-oxo-1 $H$-1,2,4-triazol-3-yl)benzenesulfonamides 26-40 when an excess of three molar equivalents of tosyl isocyanate was applied in the reaction with the corresponding aminoguanidines 11-25 in anhydrous tetrahydrofuran (THF) for at least $9 \mathrm{~h}$ at reflux. It is noteworthy, however, when 2 equivalents of tosyl isocyanate were used, no cyclization product was observed and a complex mixture of products was formed, even after considerable extending of the reaction time.

The structure of the new compounds was confirmed by elemental analyses $(\mathrm{C}, \mathrm{H}, \mathrm{N})$ and spectral (NMR, IR, MS) data presented in the experimental section. Moreover, X-ray analysis was undertaken to confirm proposed structures on the representative compound 31, which crystallized as pyridinium salt (further specified as 31Pyr, Figs. 2 and 3).

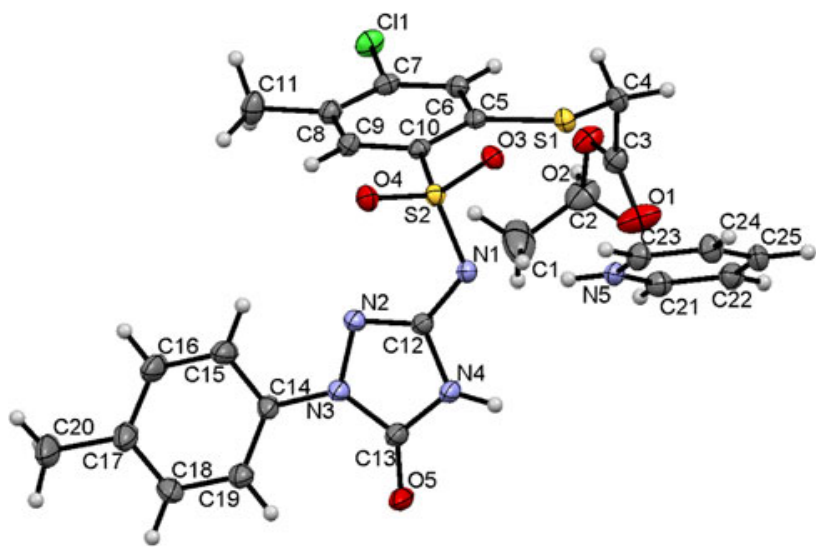

Fig. 2 Molecular structure of 31Pyr showing the atom-labeling scheme. Displacement ellipsoids drawn at the $50 \%$ probability level, with the solvating water molecule omitted

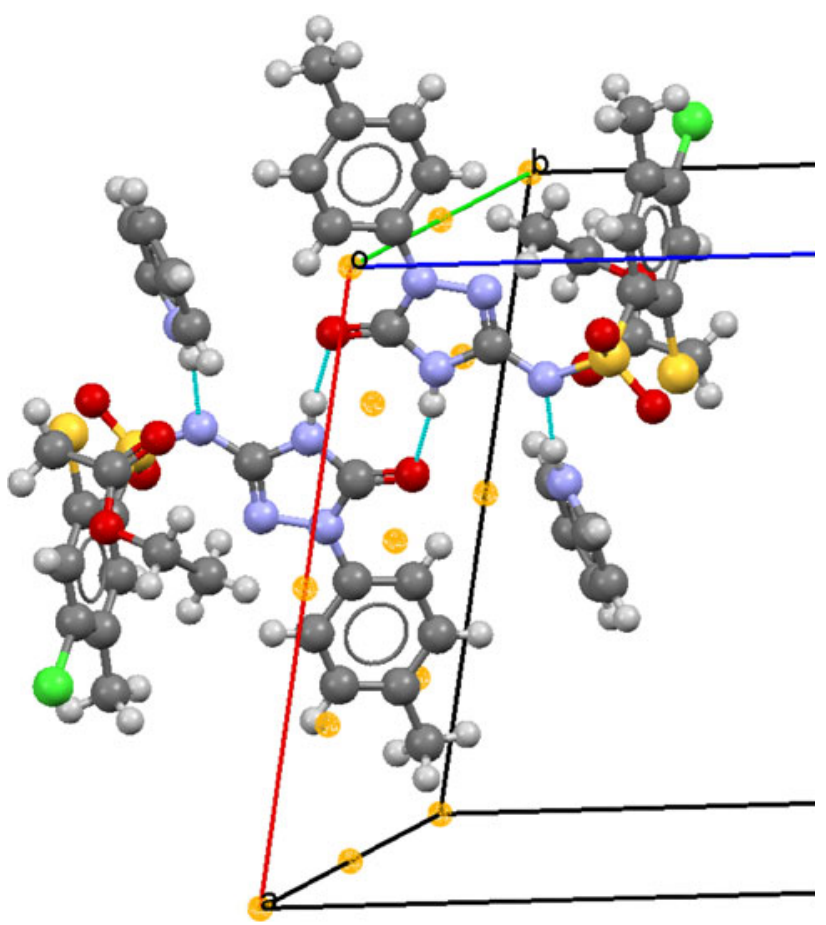

Fig. 3 Hydrogen bonds in structure of 31Pyr. Blue lines represent hydrogen bonds; transparent yellow balls denote inversion centers in the crystal (generated using Mercury CSD 2.4 [46])

\section{Molecular structure}

Details on data collection, structure solution, and refinement are given in Table 1 . Compound $\mathbf{3 1 P y r}$ crystallizes in the monoclinic space group $C 2 / c$ with (typical for this symmetry) eight molecules in the unit cell. The molecule, being a secondary benzenesulfonamide, is deprotonated at the $\mathrm{N} 1$ atom and in the crystal structure is present in the anionic form (Fig. 2). The proton is accepted by pyridine so a pyridinium ion acts as a counterion. Additionally the 
Table 1 Crystal data and structure refinement for compound 31Pyr

\begin{tabular}{|c|c|}
\hline Empirical formula & $\mathrm{C}_{20} \mathrm{H}_{20} \mathrm{ClN}_{4} \mathrm{O}_{5} \mathrm{~S}_{2} \cdot \mathrm{C}_{5} \mathrm{H}_{6} \mathrm{~N} \cdot 0.08(\mathrm{O})$ \\
\hline Formula weight & 577.42 \\
\hline Temperature/K & $120(2)$ \\
\hline Wavelength/A & 0.71073 \\
\hline Crystal system & Monoclinic \\
\hline Space group & $C 2 / c$ \\
\hline \multicolumn{2}{|l|}{ Unit cell dimensions } \\
\hline$a / \AA$ & $14.1490(3)$ \\
\hline$b / \AA$ & $14.0574(4)$ \\
\hline$c / \AA$ & $28.1211(6)$ \\
\hline$\alpha /^{\circ}$ & 90 \\
\hline$\beta /^{\circ}$ & $102.107(2)$ \\
\hline$\gamma / /^{\circ}$ & 90 \\
\hline Volume $/ \AA^{3}$ & $5,468.8(2)$ \\
\hline$Z$ & 8 \\
\hline Density (calculated) $/ \mathrm{mg} \mathrm{m}^{-3}$ & 1.403 \\
\hline Absorption coefficient $/ \mathrm{mm}^{-1}$ & 0.338 \\
\hline $\mathrm{F}(000)$ & 2405 \\
\hline Crystal size $/ \mathrm{mm}^{3}$ & $0.20 \times 0.13 \times 0.10$ \\
\hline$\theta$ range for data collection $/^{\circ}$ & $2.3-26.0$ \\
\hline Index ranges & $\begin{array}{l}-17 \leq \mathrm{h} \leq 17,-12 \leq \mathrm{k} \leq 17 \\
-25 \leq 1 \leq 34\end{array}$ \\
\hline Reflections collected & 12,491 \\
\hline Independent reflections & $5,374[R($ int $)=0.02]$ \\
\hline Completeness to $\theta=26.0^{\circ}$ & $99.6 \%$ \\
\hline Absorption correction & Numerical \\
\hline Refinement method & Full-matrix least-squares on $F^{2}$ \\
\hline Data/restraints/parameters & $5,374 / 0 / 349$ \\
\hline Goodness-of-fit on $F^{2}$ & 1.04 \\
\hline Final $R$ indices $[I>2 \sigma(I)]$ & $R_{1}=0.0405, \mathrm{w} R^{2}=0.1015$ \\
\hline$R$ indices (all data) & $R_{1}=0.0469, \mathrm{w} R^{2}=0.1074$ \\
\hline Largest diff. peak and hole/e $\cdot \AA^{-3}$ & 0.43 and -0.31 \\
\hline
\end{tabular}

solid contains solvating molecules of water that reside on twofold rotation axes, and these positions are not fully occupied by them (s.o.f. $=0.079$ ). Actually, only ca. $1 / 8$ of the $\mathrm{H}_{2} \mathrm{O}$ molecules suffice to fit to the observed electron density in this region.

The two ions are linked by a charge-assisted hydrogen bond of the $(+) \mathrm{NH} \cdots \mathrm{N}(-)$ type; pyridinium $\mathrm{N}(5)$ is a donor, and sulfonamide $\mathrm{N}(1)$ is an acceptor. Bonds $\mathrm{N}(4)-$ $\mathrm{H}(4)$ interact with carbonyl oxygen atoms $\mathrm{O} 5$ from the triazolone moiety of the neighboring molecules forming intermolecular hydrogen bonds $\mathrm{NH} \cdots \mathrm{O}$. These interactions arranged in pairs can be described by the $\mathrm{R}_{2}^{2}(8)$ motifs situated about local inversion centers (see Fig. 3). Detailed information on hydrogen bonds is given in Table 2. Packing of molecules in the solid state is reinforced also by
Table 2 Hydrogen bond geometry in crystal structure of 31Pyr

\begin{tabular}{lllll}
\hline $\mathrm{D}-\mathrm{H} \cdots \mathrm{A}$ & $\mathrm{D}-\mathrm{H} / \AA$ & $\mathrm{H} \cdots \mathrm{A} / \AA$ & $\mathrm{D} \cdots \mathrm{A} / \AA$ & $\mathrm{D}-\mathrm{H} \cdots \mathrm{A} /{ }^{\circ}$ \\
\hline $\mathrm{N} 4-\mathrm{H} 4 \cdots \mathrm{O}^{\mathrm{i}}$ & 0.88 & 1.89 & $2.765(2)$ & 171 \\
$\mathrm{~N} 5-\mathrm{H} 5 \cdots \mathrm{N} 1$ & 0.88 & 1.90 & $2.767(2)$ & 169 \\
\hline
\end{tabular}

Symmetry code: (i) $-x+1 / 2,-y+1 / 2,-z$

Table 3 Main $\pi-\pi$ stacking interaction geometry in crystal structure of 31Pyr

\begin{tabular}{|c|c|c|c|}
\hline $\operatorname{Cg}(1) \cdots \operatorname{Cg}\left(1^{\mathrm{ii}}\right) \mathrm{a} / \AA$ & $\alpha^{\mathrm{b} /{ }^{\circ}}$ & $\beta^{\mathrm{c}} /^{\circ}$ & Perp. ${ }^{\mathrm{d}}$ \\
\hline $3.8513(10)$ & 17 & 15.5 & $3.7104(7$ \\
\hline \multicolumn{4}{|c|}{ Ring (1) is composed from $\mathrm{C} 5-\mathrm{C} 10$ carbon atoms } \\
\hline \multicolumn{4}{|c|}{ Symmetry code: (ii) $-x, y, 1 / 2-z$} \\
\hline \multicolumn{4}{|c|}{${ }^{a}$ Distance between centroids } \\
\hline \multicolumn{4}{|c|}{$\mathrm{b}$ Dihedral angle between the rings } \\
\hline \multicolumn{4}{|c|}{$\begin{array}{l}\text { c Angle between the vector span on the centroids and normal to } \\
\text { ring(1) }\end{array}$} \\
\hline
\end{tabular}

$\pi-\pi$ stacking interactions between adjacent aromatic rings C5-C10 whose centers of gravity (Cg or centroids) are distant at 3.8513(10) $\AA$. The geometry of the interaction is more precisely characterized in Table 3 .

\section{Biological assay}

Compounds 27, 28, and 30-39 were initially tested at a single dose $\left(10^{-5} \mathrm{M}\right)$ in the full NCI-60 cell panel, and the results are shown in Table 4. The methodology of the in vitro cancer screen is described at the website http://www.dtp.nci.nih. gov/branches/btb/ivclsp.html.

The relatively highest sensitivity to the compounds described here was found for the cell lines of non-small cell lung cancer NCI-H522 cell line to compounds 27, 28, 31, and 38 (46 \% < IGP $<84 \%)$, leukemia RPMI-8226 to compounds 27, 28, 30, and $31(36 \%<$ IGP $<45 \%)$, HL-60(TB) to 30, 31, 36, and 39 (22\% < IGP < $91 \%)$, and K-562 to compounds $\mathbf{3 0}, \mathbf{3 1}$, and $\mathbf{3 8}(37 \%<$ IGP $<66 \%)$ as well as breast MCF7 to 30, 36, and 38 (38 \% < IGP < $83 \%$ ) (Table 4).

The following conclusions can be drawn from the structure-activity relationship study (Table 4):

1. The susceptibility of the non-small cell lung NCI-H522 cell line against 2-(benzylthio)- $N$-(2,5-dihydro-5-oxo-1$\mathrm{R}^{2}$-1H-1,2,4-triazol-3-yl)benzenesulfonamide derivatives $(\mathbf{2 7}, \mathbf{2 8}, \mathbf{3 0})$ was remarkable and increased when the methyl group $\left(\mathrm{R}^{2}=\mathrm{Me}, \quad 27, \quad\right.$ IGP $\left.=46 \%\right)$ was replaced by aromatic moieties such as phenyl $\left(\mathrm{R}^{2}=\mathrm{Ph}\right.$, 28, IGP $=69 \%)$ or tosyl $\left(\mathrm{R}^{2}=4-\mathrm{MePhSO}_{2}, \mathbf{3 0}\right.$, IGP $=71 \%$ ). The compounds mentioned above showed similar potency for RPMI-8226 $(\mathbf{2 7}, \mathrm{IGP}=36 \% ; \mathbf{2 8}$, 
Table 4 Inhibition growth percent (IGP [\%]). One-dose screening data of in vitro tumor growth inhibition for compounds $\mathbf{2 7}, \mathbf{2 8}, \mathbf{3 0}-\mathbf{3 9}$ at a dose of $10 \mu \mathrm{M}$

\begin{tabular}{|c|c|c|c|c|c|c|c|c|c|c|c|c|c|}
\hline \multirow[t]{2}{*}{ Panel } & \multirow[t]{2}{*}{ Cell line } & \multicolumn{12}{|c|}{ Compound } \\
\hline & & 27 & 28 & 30 & 31 & 32 & 33 & 34 & 35 & 36 & 37 & 38 & 39 \\
\hline Non-small cell & A549/ATCC & 26 & 62 & $*$ & $*$ & 3 & $*$ & 2 & 3 & 58 & $*$ & 5 & $*$ \\
\hline \multirow[t]{2}{*}{ Lung cancer } & NCI-H522 & 46 & 69 & 71 & 84 & NT & 5 & 5 & $*$ & NT & $*$ & 83 & $*$ \\
\hline & HOP-92 & 21 & 6 & 29 & 18 & NT & NT & 20 & NT & NT & 5 & 19 & NT \\
\hline \multirow[t]{5}{*}{ Leukemia } & RPMI-8226 & 36 & 45 & 45 & 39 & NT & 7 & 13 & 11 & NT & 7 & $*$ & $*$ \\
\hline & SR & 7 & 28 & 57 & 31 & NT & NT & 19 & NT & NT & NT & 78 & NT \\
\hline & HL-60(TB) & 19 & 13 & 27 & 26 & $*$ & 15 & $*$ & NT & 91 & $*$ & $*$ & 22 \\
\hline & MOLT-4 & 2 & 10 & 22 & 16 & $*$ & 7 & 7 & 22 & 52 & $*$ & 21 & 17 \\
\hline & K-562 & 14 & 17 & 49 & 37 & NT & NT & $*$ & NT & NT & 9 & 66 & NT \\
\hline \multirow[t]{2}{*}{ Renal cancer } & RXF 393 & 2 & 46 & $*$ & 1 & $*$ & $*$ & $*$ & $*$ & 73 & 2 & 16 & $*$ \\
\hline & UO-31 & 15 & 18 & 14 & 26 & 3 & 3 & 11 & 10 & 36 & 24 & 23 & 11 \\
\hline \multirow[t]{3}{*}{ CNS cancer } & SNB-75 & 23 & $*$ & 15 & 18 & 7 & 2 & 12 & 10 & 61 & 4 & 19 & $*$ \\
\hline & SF-295 & $*$ & $*$ & $*$ & $*$ & $*$ & 4 & 1 & 2 & 77 & 8 & 3 & $*$ \\
\hline & SF-539 & $*$ & $*$ & $*$ & $*$ & $*$ & $*$ & $*$ & $*$ & 49 & $*$ & $*$ & $*$ \\
\hline \multirow[t]{7}{*}{ Colon cancer } & HCC-2998 & 1 & $*$ & $*$ & $*$ & $*$ & $*$ & $*$ & $*$ & 41 & $*$ & 66 & 4 \\
\hline & HCT-116 & 3 & 6 & 6 & $*$ & $*$ & 1 & $*$ & 4 & 75 & $*$ & 62 & $*$ \\
\hline & HCT-15 & 4 & $*$ & 7 & $*$ & $*$ & 5 & $*$ & 4 & 65 & 1 & 38 & 2 \\
\hline & HT29 & $*$ & $*$ & 4 & $*$ & $*$ & $*$ & 1 & $*$ & 88 & $*$ & 53 & $*$ \\
\hline & SW-620 & 1 & $*$ & 9 & 5 & $*$ & 6 & 4 & $*$ & 68 & 2 & 55 & $*$ \\
\hline & COLO 205 & $*$ & $*$ & $*$ & $*$ & $*$ & NT & $*$ & NT & 71 & $*$ & NT & NT \\
\hline & KM12 & $*$ & $*$ & 5 & $*$ & $*$ & $*$ & $*$ & $*$ & 79 & $*$ & 1 & $*$ \\
\hline \multirow[t]{4}{*}{ Breast cancer } & MCF7 & $*$ & $*$ & 38 & $*$ & 2 & $*$ & 2 & $*$ & 83 & 3 & 51 & $*$ \\
\hline & $\mathrm{T}-47 \mathrm{D}$ & 8 & 3 & 20 & 9 & * & $*$ & $*$ & 1 & 31 & 6 & 41 & 1 \\
\hline & MDA-MB-468 & NT & NT & NT & NT & $*$ & NT & 3 & NT & $3^{\mathrm{a}}$ & NT & 61 & $*$ \\
\hline & HS 578T & 11 & $*$ & 24 & 5 & * & NT & 2 & NT & 46 & 12 & $*$ & NT \\
\hline \multirow[t]{2}{*}{ Ovarian cancer } & OVCAR-3 & $*$ & 1 & 8 & $*$ & $*$ & $*$ & 1 & $*$ & $5^{\mathrm{a}}$ & $*$ & 65 & \\
\hline & NCI/ADR-RES & NT & NT & NT & NT & $*$ & 2 & 5 & 9 & 64 & $*$ & 32 & \\
\hline \multirow[t]{5}{*}{ Melanoma } & UACC-257 & $*$ & 33 & $*$ & $*$ & $*$ & $*$ & 4 & $*$ & 21 & $*$ & 21 & $*$ \\
\hline & UACC-62 & 3 & 7 & 15 & 7 & $*$ & 10 & $*$ & 7 & 55 & 4 & $*$ & 9 \\
\hline & MALME-3M & 5 & NT & 5 & $*$ & $*$ & 12 & 13 & 4 & 43 & 16 & 25 & 2 \\
\hline & SK-MEL-2 & $*$ & $*$ & $*$ & 9 & $*$ & $*$ & 19 & $*$ & 26 & $*$ & NT & $*$ \\
\hline & MDA-MB-435 & NT & NT & NT & NT & 1 & 9 & $*$ & 1 & $29^{\mathrm{a}}$ & $*$ & 69 & $*$ \\
\hline Prostate cancer & PC-3 & 9 & 6 & 16 & 8 & 5 & 8 & 5 & 12 & 59 & $*$ & 7 & 6 \\
\hline
\end{tabular}

Data obtained from NCI-60 DTP human tumor cell line screening

$N T$ not tested

* Not active

${ }^{\text {a }}$ Cytotoxic effect (lethality)

IGP $=45 \% ; \quad 30, \quad$ IGP $=45 \%) \quad$ and $\quad$ SR $\quad(27$, IGP $=7 \% ; \mathbf{2 8}$, IGP $=28 \% ; \mathbf{3 0}$, IGP $=57 \%$ ) lines of leukemia. It should be noted, moreover, that replacement of $\mathrm{R}^{2}=\mathrm{Ph}(\mathbf{2 8})$ for $\mathrm{R}^{2}=4-\mathrm{MePhSO}_{2}(\mathbf{3 0})$ caused loss of activity against non-small cell lung cancer (A549/ATCC) and renal (RXF 393) cell lines.

2. For the series of $\mathrm{N}$-(4,5-dihydro-5-oxo-1-phenyl- $1 \mathrm{H}$ 1,2,4-triazol-3-yl)-2-( $\mathrm{R}^{1}$-methylthio)benzenesulfonamides the substituent at the sulfur atom S-2 at the 2-position of benzenesulfonamide has an impact on the antiproliferative activity against some cancer cell lines: exchange for instance of $\mathrm{R}^{1}=1$-naphthyl in 36 into $\mathrm{R}^{1}=\mathrm{Ph}(\mathbf{2 8})$, 1,3-dioxolan-1-yl (32), 3- $\mathrm{CF}_{3} \mathrm{Ph}$ (34), 4- $\mathrm{CF}_{3} \mathrm{Ph}(\mathbf{3 5})$, and 1,2-dihydro-2-oxoquinolin-4yl (39) decreased activity against the leukemia HL60(TB) cell line, as well as the leukemia MOLT-4 cell line; replacing $\mathrm{R}^{1}=1$-naphthyl or $\mathrm{Ph}$ for $\mathrm{R}^{1}=3$ $\mathrm{CF}_{3} \mathrm{Ph}, 4-\mathrm{CF}_{3} \mathrm{Ph}$ or 1,2-dihydro-2-oxoquinolin-4-yl 
Table 5 The in vitro tumor growth inhibition data for compound $\mathbf{3 6}$

\begin{tabular}{|c|c|c|c|c|}
\hline Panel & Cell line & $\begin{array}{l}\mathrm{GI}_{50}^{\mathrm{a}} / \\
\mu \mathrm{M}\end{array}$ & $\begin{array}{l}\mathrm{TGI}^{\mathrm{b}} / \\
\mu \mathrm{M}\end{array}$ & $\begin{array}{l}\mathrm{LC}_{50}^{\mathrm{c}} / \\
\mu \mathrm{M}\end{array}$ \\
\hline \multirow[t]{5}{*}{ Leukemia } & CCRF-CEM & 3.2 & 13.2 & $>100$ \\
\hline & HL-60(TB) & 3.1 & 9.2 & 58.7 \\
\hline & K-562 & 3.6 & 13.9 & $>100$ \\
\hline & MOLT-4 & 5.7 & 37.2 & $>100$ \\
\hline & RPMI-8226 & 3.6 & 28.1 & $>100$ \\
\hline \multirow{9}{*}{$\begin{array}{l}\text { Non-small cell lung } \\
\text { cancer }\end{array}$} & A549/ATCC & 4.4 & 27.8 & $>100$ \\
\hline & EKVX & 4.6 & 23.3 & $>100$ \\
\hline & HOP-62 & 7.1 & 20.8 & 49.3 \\
\hline & HOP-92 & 7.5 & 23.0 & 57.0 \\
\hline & NCI-H226 & 3.6 & 16.1 & 53.0 \\
\hline & NCI-H23 & 3.1 & 11.7 & 42.8 \\
\hline & NCI-H322M & 8.3 & 32.9 & $>100$ \\
\hline & NCI-H460 & 2.8 & 11.7 & $>100$ \\
\hline & NCI-H522 & 2.5 & 8.3 & 44.1 \\
\hline \multirow[t]{7}{*}{ Colon cancer } & COLO 205 & 2.3 & 4.9 & 11.1 \\
\hline & HCC-2998 & 3.5 & 12.6 & 40.0 \\
\hline & НCT-116 & 3.4 & 12.6 & 43.0 \\
\hline & НCT-15 & 4.0 & 15.3 & 47.8 \\
\hline & HT29 & 3.5 & 11.7 & 41.9 \\
\hline & KM12 & 3.2 & 12.5 & 46.1 \\
\hline & SW-620 & 4.6 & 18.6 & 48.9 \\
\hline \multirow[t]{6}{*}{ CNS cancer } & SF-268 & 3.7 & 15.5 & 48.2 \\
\hline & SF-295 & 3.0 & 13.9 & $>100$ \\
\hline & SF-539 & 3.2 & 9.4 & 44.1 \\
\hline & SNB-19 & 4.8 & 23.5 & 95.3 \\
\hline & SNB-75 & 2.9 & 15.5 & 85.1 \\
\hline & $\mathrm{U} 251$ & 5.3 & 19.9 & 54.9 \\
\hline \multirow[t]{9}{*}{ Melanoma } & LOX IMVI & 4.8 & 18.3 & 48.8 \\
\hline & MALME-3 M & 8.9 & 29.4 & 92.2 \\
\hline & M14 & 3.2 & 11.5 & 64.9 \\
\hline & MDA-MB-435 & 1.9 & 5.5 & 32.0 \\
\hline & SK-MEL-2 & 5.8 & 20.7 & 60.2 \\
\hline & SK-MEL-28 & 6.0 & 20.1 & 54.2 \\
\hline & SK-MEL-5 & 3.6 & 13.1 & 36.6 \\
\hline & UACC-257 & 7.9 & 33.6 & $>100$ \\
\hline & UACC-62 & 4.0 & 16.1 & 48.5 \\
\hline \multirow[t]{7}{*}{ Ovarian cancer } & IGROV1 & 4.9 & 21.9 & 97.0 \\
\hline & OVCAR-3 & 2.4 & 6.2 & 23.6 \\
\hline & OVCAR-4 & 4.2 & 16.5 & 55.1 \\
\hline & OVCAR-5 & 6.3 & 19.4 & 46.8 \\
\hline & OVCAR-8 & 5.0 & 33.0 & $>100$ \\
\hline & NCI/ADR-RES & 2.7 & 8.6 & $>100$ \\
\hline & SK-OV-3 & 3.9 & 18.1 & $>100$ \\
\hline \multirow[t]{5}{*}{ Renal cancer } & $786-0$ & 7.9 & 22.0 & 54.3 \\
\hline & A498 & 1.9 & 10.5 & 38.0 \\
\hline & $\mathrm{ACHN}$ & 5.8 & 21.9 & 68.7 \\
\hline & CAKI-1 & 4.2 & 25.9 & $>100$ \\
\hline & RXF 393 & 2.5 & 7.6 & 32.2 \\
\hline
\end{tabular}

Table 5 continued

\begin{tabular}{lllll}
\hline Panel & Cell line & $\begin{array}{l}\mathrm{GI}_{50}^{\mathrm{a}} / \\
\mu \mathrm{M}\end{array}$ & $\begin{array}{l}\mathrm{TGI}^{\mathrm{b}} / \\
\mu \mathrm{M}\end{array}$ & $\begin{array}{l}\mathrm{LC}_{50}^{\mathrm{c}} / \\
\mu \mathrm{M}\end{array}$ \\
\hline \multirow{3}{*}{ TK-10 } & SN12C & 4.6 & 18.2 & 52.7 \\
UO-31 & 10.3 & 24.2 & 56.8 & \\
Prostate cancer & 5.5 & 18.7 & 45.9 & \\
& PC-3 & 3.6 & 17.1 & 73.7 \\
Breast cancer & DU-145 & 2.9 & 8.6 & 32.6 \\
& MCF7 & 3.8 & 15.3 & $>100$ \\
& MDA-MB-231/ & 5.6 & 20.5 & 60.4 \\
& ATCC & & & \\
& HS 578T & 2.9 & 19.6 & $>100$ \\
& BT-549 & 11.2 & 26.0 & 60.4 \\
& T-47D & 6.1 & 35.9 & $>100$ \\
& MDA-MB-468 & 3.0 & 12.3 & 49.9 \\
\hline
\end{tabular}

Data obtained from NCI-60 DTP human tumor cell line screening

${ }^{\mathrm{a}} \mathrm{GI}_{50}$ : molar concentration that inhibits $50 \%$ net cell growth

b TGI: molar concentration giving total growth inhibition

${ }^{c} \mathrm{LC}_{50}$ : molar concentration causing $50 \%$ net cell death

resulted in loss of activity against cell lines non-small cell lung A549/ATCC and renal RXF 393.

3. The significant susceptibility of almost the entire colon cancer subpanel against $N$-(4,5-dihydro-5-oxo- $1-\mathrm{R}^{2}-1 H$ 1,2,4-triazol-3-yl)-2-(naphthalen-1-ylmethylthio)benzenesulfonamides $\mathbf{3 6}$ and $\mathbf{3 8}$ should be pointed out. Moreover, the exchange of $\mathrm{R}^{2}=\mathrm{Ph}(36)$ or $4-\mathrm{MePh}$ $\mathrm{SO}_{2}(\mathbf{3 8})$ for 4-ClPh (37) led to a lack of susceptibility of HCC-2998, HTC-116, HTC-15, HT29, and SW-620.

Further anticancer evaluation was performed at five-dose assay on the distinctive compound $\mathbf{3 6}$. The anticancer activity of the tested compound was reported for each cell line by the parameters $\mathrm{GI}_{50}$ (molar concentration of the compounds that inhibit $50 \%$ net cell growth), TGI (molar concentration of the compounds leading to total inhibition), and $\mathrm{LC}_{50}$ (molar concentration of the compounds causing $50 \%$ net cell death). The susceptibility of individual subpanels indicates the following order: prostate, colon, CNS, leukemia, ovarian, nonsmall cell lung, melanoma, renal, and breast cancer (Table 5). As shown in Table 5, compound $\mathbf{3 6}$ exhibited remarkable activity at low $\mathrm{GI}_{50}$ level $<11.2 \mu \mathrm{M}\left(\mathrm{MID} \mathrm{GI}_{50}=4.2 \mu \mathrm{M}\right)$ over a number of cancer cell lines, acting effectively against 13 human tumor cell lines with $\mathrm{GI}_{50}$ values in the low micromolar range of 1.9-3.0 $\mu \mathrm{M}$ with selectivity toward melanoma MDA-MB-435 $\left(\mathrm{GI}_{50}=1.9 \mu \mathrm{M}\right.$, TGI $\left.=5.5\right)$ and renal A498 $\left(\mathrm{GI}_{50}=1.9 \mu \mathrm{M}\right.$, TGI $\left.=10.5\right)$ cell lines. It is worth mentioning that lines HL-60(TB), NCI-H522, COLO 205, SF-539, MDA-MB-435, OVCAR-3, A498, RXF 393, DU-145, and MDA-MB-468 were characterized by the 
relatively low parameters $\mathrm{GI}_{50}(1.9-3.2 \mu \mathrm{M})$, TGI (4.9-12.3 $\mu \mathrm{M})$, and $\mathrm{LC}_{50}$ below $58.7 \mu \mathrm{M}$.

A COMPARE [47] analysis at the NCI of compound $\mathbf{3 6}$ showed a moderate Pearson's correlation coefficient $(\mathrm{PCC}=0.473-0.425)$ with agents disrupting microtubule formation such as maytansine and rhizoxin [48].

\section{Conclusion}

We have developed a new method for the synthesis of a series of 2-(alkylthio)-4-chloro- $N$-(4,5-dihydro-5-oxo-1- $\mathrm{R}^{2}-1 H-1,2$, 4-triazol-3-yl)-5-methylbenzenesulfonamides 26-40. The prominent compound $\mathbf{3 6}$ showed high $\left(\mathrm{GI}_{50}=1.9-3.0 \mu \mathrm{M}\right)$ activity against 13 of the tumor cell lines and reasonable activity at level $\mathrm{GI}_{50}<11.2 \mu \mathrm{M}\left(\mathrm{MID} \mathrm{GI}_{50}=4.2 \mu \mathrm{M}\right)$ over a number cell lines, suggesting that $\mathbf{3 6}$ may be a useful lead compound for the search for more powerful anticancer agents with low toxicity against normal cells.

\section{Experimental}

The following instruments and parameters were used: melting points: Boetius apparatus; IR spectra: $\mathrm{KBr}$ pellets, 400-4,000 $\mathrm{cm}^{-1}$, Thermo Mattson Satellite FTIR spectrometer; ${ }^{1} \mathrm{H}$ NMR and ${ }^{13} \mathrm{C}$ NMR: Varian Gemini 200 apparatus or Varian Unity Plus $500 \mathrm{MHz}$, chemical shifts are expressed as $\delta$ values relative to $\mathrm{Me}_{4} \mathrm{Si}$ as standard; LC-MS analyses: Shimadzu LCMS-IT-TOF LC-20A mass spectrometer with an electrospray ionization, capillary voltage in positive ion mode $+4.5 \mathrm{kV}$, column: Jupiter $4 \mathrm{u}$ Proteo $90 \AA$, $4.0 \times 150 \mathrm{~mm}, 4 \mu \mathrm{m}$, mobile phase: A-water with $0.1 \%$ formic acid, $\mathrm{B}-0.1 \%$ formic acid in acetonitrile, linear gradient $50-100 \% \mathrm{~B}$ in $45 \mathrm{~min}$, flow rate: $0.2 \mathrm{~cm}^{3} \mathrm{~min}^{-1}$. The results of elemental analyses for $\mathrm{C}, \mathrm{H}$, and $\mathrm{N}$ were in agreement with the calculated values within $\pm 0.3 \%$ range. Thin-layer chromatography (TLC) was performed on Merck Kieselgel 60F254 plates and visualized with UV. $N-(5-$ Methylphenylsulfonyl)cyanamide potassium salts $3, \mathbf{4}$ and aminoguanidines 11-14 and $\mathbf{1 6}$ were obtained in accordance with the previously described procedures $[2,36]$.

\section{N-[4-Chloro-2-(1,3-dioxolan-2-ylmethylthio)- \\ 5-methylphenylsulfonyl]cyanamide potassium salt $\left(5, \mathrm{C}_{12} \mathrm{H}_{12} \mathrm{ClKN}_{2} \mathrm{O}_{4} \mathrm{~S}_{2}\right)$}

To a suspension of $3.05 \mathrm{~g}$ 5-chloro-2-(cyanoaminosulfonyl)-4-methylthiophenolate dipotassium salt $(2,9 \mathrm{mmol})$ in $9 \mathrm{~cm}^{3}$ methanol $2.4 \mathrm{~cm}^{3}$ 2-(bromomethyl)-1,3-dioxolane $(23 \mathrm{mmol})$ was added dropwise for $5 \mathrm{~min}$. The reaction mixture was stirred at $65^{\circ} \mathrm{C}$ for $6.5 \mathrm{~h}$, then $12 \mathrm{~h}$ at room temperature. The precipitate was collected by filtration. The filtrate was evaporated to dryness, and the residue was triturated with $90 \mathrm{~cm}^{3}$ diethyl ether to give a second fraction of precipitate. The product was extracted from the combined fractions of solid with hot ethanol to give $2.99 \mathrm{~g}(86 \%) \mathbf{5}$. M.p.: $224-225{ }^{\circ} \mathrm{C}$; TLC: $R_{\mathrm{f}}=0.74\left(\mathrm{CHCl}_{3}: \mathrm{MeOH}=3: 1\right)$; IR (KBr): $\bar{v}=2,924\left(\mathrm{CH}_{3}, \mathrm{CH}_{2}\right), 2,854\left(\mathrm{CH}_{3}, \mathrm{CH}_{2}\right), 2,179$ $(\mathrm{C} \equiv \mathrm{N}), 1,339,1,145\left(\mathrm{SO}_{2}\right) \mathrm{cm}^{-1} ;{ }^{1} \mathrm{H}$ NMR $(200 \mathrm{MHz}$, DMSO- $\left.d_{6}\right): \delta=2.31\left(\mathrm{~s}, 3 \mathrm{H}, \mathrm{CH}_{3}\right), 3.21\left(\mathrm{~d}, 2 \mathrm{H}, \mathrm{S}-\mathrm{CH}_{2}\right)$, 3.79-4.00 (m, 4H, $\left.\mathrm{CH}_{2}-\mathrm{O}\right), 5.11(\mathrm{t}, 1 \mathrm{H}, \mathrm{CH}-\mathrm{O}), 7.46(\mathrm{~s}, 1 \mathrm{H}$, $\mathrm{H}-3), 7.77$ (s, 1H, H-6) ppm; ${ }^{13} \mathrm{C}$ NMR (50 MHz, DMSO$\left.d_{6}\right): \delta=19.22,36.04,64.96,102.34,117.50,127.42$, $130.74,131.36,135.75,135.97,140.86 \mathrm{ppm}$.

General procedure for the preparation of N-[4-chloro5-methyl-2-( $R^{1}$-methylthio)phenylsulfonyl]cyanamide potassium salts $\mathbf{6}-\mathbf{1 0}$

To a suspension of $3.05 \mathrm{~g}$ 5-chloro-2-(cyanoaminosulfonyl)-4-methylthiophenolate dipotassium salt $(2,9 \mathrm{mmol})$ in methanol or ethanol the appropriate halomethyl electrophile was added. The reaction mixture was stirred at room temperature or at $65{ }^{\circ} \mathrm{C}$. The precipitate was collected by filtration. The product was separated from inorganic salts by extraction with $200 \mathrm{~cm}^{3}$ hot ethanol.

N-[4-Chloro-5-methyl-2-[3-(trifluoromethyl)benzylthio]phenylsulfonyl]cyanamide potassium salt $\left(6, \mathrm{C}_{16} \mathrm{H}_{11} \mathrm{ClF}_{3} \mathrm{KN}_{2} \mathrm{O}_{2} \mathrm{~S}_{2}\right)$

Starting from 2 in $45 \mathrm{~cm}^{3}$ ethanol and $1.3 \mathrm{~cm}^{3}$ 3-(trifluoromethyl)benzyl chloride ( $9 \mathrm{mmol}$ ) for $2 \mathrm{~h}$ at room temperature, compound 6 was obtained. Yield: $3.46 \mathrm{~g}(84 \%)$; m.p.: 158 $-160{ }^{\circ} \mathrm{C}$; TLC: $R_{\mathrm{f}}=0.87\left(\mathrm{CHCl}_{3}: \mathrm{MeOH}=2: 1\right)$; IR $(\mathrm{KBr})$ : $\bar{v}=2,924\left(\mathrm{CH}_{3}, \mathrm{CH}_{2}\right), 2,174(\mathrm{C} \equiv \mathrm{N}), 1,332,1,132\left(\mathrm{SO}_{2}\right)$ $\mathrm{cm}^{-1} ;{ }^{1} \mathrm{H}$ NMR $\left(200 \mathrm{MHz}, \mathrm{DMSO}-d_{6}\right): \delta=2.31\left(\mathrm{~s}, 3 \mathrm{H}, \mathrm{CH}_{3}\right)$, $4.41\left(\mathrm{~s}, 2 \mathrm{H}, \mathrm{S}-\mathrm{CH}_{2}\right), 7.41(\mathrm{~s}, 1 \mathrm{H}, \mathrm{H}-3), 7.58-7.62(\mathrm{~m}, 2 \mathrm{H}, \mathrm{Ar})$, 7.76-7.81 (m, 3H, H-6, Ar) ppm; ${ }^{13} \mathrm{C}$ NMR (50 MHz, DMSO$\left.d_{6}\right): \delta=19.23,35.73,117.46,124.12,124.20,125.95,126.03$, $127.78,129.77,130.85,131.89,133.49,134.82,135.94$, 138.57, $141.12 \mathrm{ppm}$.

N-[4-Chloro-5-methyl-2-[4-(trifluoromethyl)benzylthio]phenylsulfonyl]cyanamide potassium salt $\left(7, \mathrm{C}_{16} \mathrm{H}_{11} \mathrm{ClF}_{3} \mathrm{KN}_{2} \mathrm{O}_{2} \mathrm{~S}_{2}\right)$

Starting from 2 in $45 \mathrm{~cm}^{3}$ ethanol and $1.3 \mathrm{~cm}^{3}$ 4-(trifluoromethyl)benzyl chloride $(9 \mathrm{mmol})$ for $4 \mathrm{~h}$ at room temperature, compound 7 was obtained. Yield: 3.64 g (88 \%); m.p.: 177 $-178{ }^{\circ} \mathrm{C}$; TLC: $R_{\mathrm{f}}=0.69$ (ethyl acetate:isopropanol $=2: 1$ ); IR (KBr): $\bar{v}=2,921\left(\mathrm{CH}_{3}, \mathrm{CH}_{2}\right), 2,176(\mathrm{C} \equiv \mathrm{N}), 1,327,1,137$ $\left(\mathrm{SO}_{2}\right) \mathrm{cm}^{-1} ;{ }^{1} \mathrm{H}$ NMR (500 MHz, DMSO- $\left.d_{6}\right): \delta=2.29$ (s, 3H, $\left.\mathrm{CH}_{3}\right), 4.38\left(\mathrm{~s}, 2 \mathrm{H}, \mathrm{S}-\mathrm{CH}_{2}\right), 7.38(\mathrm{~s}, 1 \mathrm{H}, \mathrm{H}-3), 7.66(\mathrm{~d}$, 2H, Ar), 7.68 (d, 2H, Ar), 7.73 (s, 1H, H-6) ppm; ${ }^{13} \mathrm{C} \mathrm{NMR}$ $\left(50 \mathrm{MHz}, \mathrm{DMSO}-d_{6}\right): \delta=19.23,35.78,117.50,125.41,125$. 49, 125.56, 127.57, 130.13, 130.87, 131.84, 134.93, 135.96, $141.06,142.02 \mathrm{ppm}$. 
N-[4-Chloro-5-methyl-2-(naphthalen-1-ylmethylthio)phenylsulfonyl]cyanamide potassium

salt $\left(\mathbf{8}, \mathrm{C}_{19} \mathrm{H}_{14} \mathrm{ClKN}_{2} \mathrm{O}_{2} \mathrm{~S}_{2}\right)$

Starting from 2 in $10 \mathrm{~cm}^{3}$ ethanol and $1.3 \mathrm{~cm}^{3} 1$-(chloromethyl)naphthalene $(9 \mathrm{mmol})$ for $1 \mathrm{~h}$ at room temperature, compound 8 was obtained. Yield: $3.09 \mathrm{~g}$ (78 \%); m.p.: 223-225 ${ }^{\circ} \mathrm{C}$; TLC: $R_{\mathrm{f}}=0.63$ (ethyl acetate:isopropanol $=2: 1) ; \quad$ IR $\quad(\mathrm{KBr}): \bar{v}=2,922 \quad\left(\mathrm{CH}_{3}, \mathrm{CH}_{2}\right), \quad 2,175$ $(\mathrm{C} \equiv \mathrm{N}), 1,341,1,140\left(\mathrm{SO}_{2}\right) \mathrm{cm}^{-1} ;{ }^{1} \mathrm{H} \mathrm{NMR}(500 \mathrm{MHz}$, DMSO- $\left.d_{6}\right): \delta=2.32\left(\mathrm{~s}, 3 \mathrm{H}, \mathrm{CH}_{3}\right), 4.72\left(\mathrm{~s}, 2 \mathrm{H}, \mathrm{S}-\mathrm{CH}_{2}\right)$, 7.44-7.47 (m, 1H, Ar), $7.51(\mathrm{~s}, 1 \mathrm{H}, \mathrm{H}-3), 7.52-7.59(\mathrm{~m}$, 2H, Ar), 7.61 (d, 1H, Ar), $7.76(\mathrm{~s}, 1 \mathrm{H}, \mathrm{H}-6), 7.87$ (d, 1H, Ar), 7.95 (d, 1H, Ar), 8.24 (d, 1H, Ar) ppm; ${ }^{13} \mathrm{C}$ NMR $\left(50 \mathrm{MHz}, \mathrm{DMSO}-d_{6}\right): \delta=19.29,34.68,117.49,124.62$, $125.78,126.20,126.52,127.76,128.20,128.36,128.76$, $130.80,131.60,131.71,132.20,133.68,136.04,136.15$, $140.76 \mathrm{ppm}$.

N-[4-Chloro-2-(1,2-dihydro-2-oxoquinolin-4-ylmethylthio)-5-methylphenylsulfonyl]cyanamide potassium salt $\left(\mathbf{9}, \mathrm{C}_{18} \mathrm{H}_{13} \mathrm{ClKN}_{3} \mathrm{O}_{3} \mathrm{~S}_{2}\right)$

Starting from 2 in $42 \mathrm{~cm}^{3}$ ethanol and $2.1 \mathrm{~g} \mathrm{4-(bromo-}$ methyl)quinolin-2(1H)-one $(9 \mathrm{mmol})$ for $4 \mathrm{~h}$ at room temperature, compound 9 was obtained. Yield: $3.60 \mathrm{~g}$ (88\%); m.p.: 199-201 ${ }^{\circ}$ C; TLC: $R_{\mathrm{f}}=0.61$ (ethyl acetate:isopropanol:acetic acid $=1: 1: 0.02) ; \operatorname{IR~}(\mathrm{KBr}): \bar{v}=$ 2,922 $\left(\mathrm{CH}_{3}, \mathrm{CH}_{2}\right), 2,181(\mathrm{C} \equiv \mathrm{N}), 1,668(\mathrm{CO}), 1,341,1,142$ $\left(\mathrm{SO}_{2}\right) \mathrm{m}^{-1} ;{ }^{1} \mathrm{H}$ NMR $\left(500 \mathrm{MHz}, \mathrm{DMSO}-d_{6}\right): \delta=2.31(\mathrm{~s}$, $\left.3 \mathrm{H}, \mathrm{CH}_{3}\right), 4.52\left(\mathrm{~s}, 2 \mathrm{H}, \mathrm{S}-\mathrm{CH}_{2}\right), 6.57(\mathrm{~s}, 1 \mathrm{H}, \mathrm{Ar}), 7.21(\mathrm{t}$, 1H, Ar), 7.32 (d, 1H, Ar), 7.40 (s, 1H, H-3), 7.51 (t, 1H, Ar), 7.77 (s, 1H, H-6), 7.93 (d, 1H, Ar), 11.74 (s, 1H, $\mathrm{NH}) \mathrm{ppm} ;{ }^{13} \mathrm{C}$ NMR $\left(50 \mathrm{MHz}, \mathrm{DMSO}-d_{6}\right): \delta=19.30$, $33.33,115.88,117.50,118.47,121.99,122.08,125.42$, $128.14,130.82,132.21,134.60,136.04,139.22,141.24$, 146.29, 161.59 ppm.

N-[4-Chloro-2-(2,3-dihydrobenzo[b][1,4]dioxin-2ylmethylthio)-5-methylphenylsulfonyl]cyanamide potassium salt $\left(\mathbf{1 0}, \mathrm{C}_{17} \mathrm{H}_{14} \mathrm{ClKN}_{2} \mathrm{O}_{4} \mathrm{~S}_{2}\right)$

Starting from 2 in $23 \mathrm{~cm}^{3}$ methanol and $1.7 \mathrm{~cm}^{3}$ 2-(bromomethyl)-1,4-benzodioxane $(12 \mathrm{mmol})$ for $6 \mathrm{~h}$ at $65^{\circ} \mathrm{C}$, compound 10 was obtained. Yield: $3.2 \mathrm{~g}$ (78 \%); m.p.: 98-100 ${ }^{\circ} \mathrm{C}$; TLC: $R_{\mathrm{f}}=0.86\left(\mathrm{CHCl}_{3}\right.$ :pentane:acetone $=$ 1:1:0.5); IR (KBr): $\bar{v}=2,923\left(\mathrm{CH}_{3}, \mathrm{CH}_{2}\right), 2,176(\mathrm{C} \equiv \mathrm{N})$, $1,343,1,143\left(\mathrm{SO}_{2}\right) \mathrm{cm}^{-1} ;{ }^{1} \mathrm{H}$ NMR (200 MHz, DMSO- $\left.d_{6}\right)$ : $\delta=2.33\left(\mathrm{~s}, 3 \mathrm{H}, \mathrm{CH}_{3}\right), 3.34\left(\mathrm{~d}, 2 \mathrm{H}, \mathrm{S}-\mathrm{CH}_{2}\right), 4.04-4.13(\mathrm{~m}$, 2H, $\mathrm{CH}_{2}-\mathrm{O}$ ), 4.29-4.43 (m, 1H, CH-O), 6.80-6.91 (m, 4H, Ar), 7.57 (s, 1H, H-3), 7.78 (s, 1H, H-6) ppm; ${ }^{13} \mathrm{C}$ NMR $\left(50 \mathrm{MHz}, \mathrm{DMSO}-d_{6}\right): \delta=19.30,32.96,66.34,71.91$, $107.38,117.23,117.37,121.61,121.79,128.34,130.85$, $132.24,134.60,136.20,141.72,142.81,143.13$ ppm.
General procedure for the preparation of 1-[4-chloro5-methyl-2-( $R^{1}$-methylthio)phenylsulfonyl]-3$\left(R^{2}\right.$-amino $)$ guanidines $15,17-25$

To a suspension of the appropriate $N$-(phenylsulfonyl)cyanamide potassium salt $(\mathbf{3}, \mathbf{5}-\mathbf{1 0}, 3.5 \mathrm{mmol})$ in dry toluene was added the corresponding phenylhydrazine hydrochloride derivative $(3.5 \mathrm{mmol})$ or $p$-toluenesulfonyl hydrazide $(3.5 \mathrm{mmol})$ in the presence of $p$-toluenesulfonic acid monohydrate (PTSA, $3.5 \mathrm{mmol}$ ). The reaction mixture was stirred at reflux for $1-8 \mathrm{~h}$, and left overnight at $0{ }^{\circ} \mathrm{C}$. The precipitate was filtered off, and dried, then treated with $20 \mathrm{~cm}^{3}$ of water. After vigorously stirring for $30 \mathrm{~min}$ the precipitate was collected by filtration, dried, and crystallized from ethanol $(\mathbf{1 5}, \mathbf{1 7}, \mathbf{1 9}, \mathbf{2 1 - 2 3}, \mathbf{2 5})$, ethyl acetate/ hexane $(\mathbf{1 8})$, or ethyl acetate $(\mathbf{2 0}, \mathbf{2 4})$.

1-[2-(Benzylthio)-4-chloro-5-methylphenylsulfonyl]-3(4-methylphenylsulfonylamino)guanidine

$\left(15, \mathrm{C}_{22} \mathrm{H}_{23} \mathrm{ClN}_{4} \mathrm{O}_{4} \mathrm{~S}_{3}\right)$

Method A. According to the general procedure, starting from $1.37 \mathrm{~g} \mathrm{3}, 0.65 \mathrm{~g} p$-toluenesulfonyl hydrazide, and $0.66 \mathrm{~g}$ PTSA in $40 \mathrm{~cm}^{3}$ of dry toluene for $1 \mathrm{~h}$, the title compound 15 was obtained. Yield: $1.62 \mathrm{~g}$ (86 \%); m.p.: $242-244{ }^{\circ} \mathrm{C}$; TLC: $\quad R_{\mathrm{f}}=0.38 \quad\left(\mathrm{CHCl}_{3}\right.$ :pentane:acetone $\left.=1: 1: 0.5\right) ;$ IR $(\mathrm{KBr}): \bar{v}=3,469,3,361(\mathrm{NH}), 2,922,2,832\left(\mathrm{CH}_{3}, \mathrm{CH}_{2}\right)$, 1,384, 1,340, 1,172, 1,141 $\left(\mathrm{SO}_{2}\right) \mathrm{cm}^{-1} ;{ }^{1} \mathrm{H} \mathrm{NMR}(500 \mathrm{MHz}$, DMSO- $\left.d_{6}\right): \delta=2.30\left(\mathrm{~s}, 3 \mathrm{H}, \mathrm{CH}_{3}\right), 2.39$ (s, 3H, $\left.\mathrm{CH}_{3}\right), 4.29$ (s, 2H, S- $\mathrm{CH}_{2}$ ), 7.18 (brs, 1H, NH=), 7.25-7.28 (m, 1H, Ar), 7.32-7.38 (m, 4H, Ar), 7.42-7.43 (m, 4H, H-3, NH, Ar), 7.66 (d, 2H, Ar), 7.79 (s, 1H, H-6), 9.23 (s, 1H, N-NHSO 2 ), 9.89 (s, $\left.1 \mathrm{H}, \mathrm{SO}_{2} \mathrm{NH}\right) \mathrm{ppm} ;{ }^{13} \mathrm{C} \mathrm{NMR}\left(50 \mathrm{MHz}, \mathrm{DMSO}-d_{6}\right)$ : $\delta=19.20,21.35,36.60,127.51,128.04,128.15,128.74$, $129.41,129.89,130.58,132.09,134.71,135.86,136.48$, 136.74, 139.64, 144.17, 158.47 ppm.

Method B. To a cooled mixture of $1.35 \mathrm{~g} 11$ (3.5 mmol) in $5 \mathrm{~cm}^{3}$ dry pyridine was added $0.67 \mathrm{~g}$ tosyl chloride ( $3.5 \mathrm{mmol})$. The ice bath was removed and the mixture was stirred at room temperature for $4 \mathrm{~h}$, then at $60-65{ }^{\circ} \mathrm{C}$ for $5 \mathrm{~h}$. After standing overnight, the mixture was added dropwise to $12 \mathrm{~cm}^{3}$ slush and vigorously stirred for $2 \mathrm{~h}$. The solid was filtered off, washed with water $\left(5 \times 20 \mathrm{~cm}^{3}\right)$, $1 \% \mathrm{HCl}\left(2 \times 20 \mathrm{~cm}^{3}\right)$, water $\left(2 \times 20 \mathrm{~cm}^{3}\right)$ and dried. Purification from $\mathrm{MeOH}$ yielded 15 (86\%); m.p. 242-244 ${ }^{\circ} \mathrm{C}$ (dec.); IR and ${ }^{1} \mathrm{H}$ NMR spectra were identical with an authentic sample of $\mathbf{1 5}$.

1-[4-Chloro-2-(1,3-dioxolan-2-ylmethylthio)-

5-methylphenylsulfonyl]-3-(phenylamino)guanidine (17, $\left.\mathrm{C}_{18} \mathrm{H}_{21} \mathrm{ClN}_{4} \mathrm{O}_{4} \mathrm{~S}_{2}\right)$

Starting from $1.36 \mathrm{~g} 5$ and $0.51 \mathrm{~g}$ phenylhydrazine hydrochloride in $5 \mathrm{~cm}^{3}$ dry toluene for $1 \mathrm{~h}$, the title compound 
17 was obtained. Yield: $0.80 \mathrm{~g}(50 \%)$; m.p.: $173-175{ }^{\circ} \mathrm{C}$; TLC: $R_{\mathrm{f}}=0.59\left(\mathrm{CHCl}_{3}:\right.$ pentane:acetone $\left.=1: 1: 0.5\right) ;$ IR $(\mathrm{KBr}): \bar{v}=3,447(\mathrm{NH}), 2,923\left(\mathrm{CH}_{3}, \mathrm{CH}_{2}\right), 1,393,1140$ $\left(\mathrm{SO}_{2}\right) \mathrm{cm}^{-1} ;{ }^{1} \mathrm{H}$ NMR $\left(500 \mathrm{MHz}, \mathrm{DMSO}-d_{6}\right): \delta=2.32(\mathrm{~s}$, $\left.3 \mathrm{H}, \mathrm{CH}_{3}\right), 3.28\left(\mathrm{~d}, 2 \mathrm{H}, \mathrm{S}-\mathrm{CH}_{2}\right), 3.79-3.85\left(\mathrm{~m}, 2 \mathrm{H}, \mathrm{CH}_{2}-\mathrm{O}\right)$, 3.92-3.98 (m, 2H, $\left.\mathrm{CH}_{2}-\mathrm{O}\right), 5.14(\mathrm{t}, 1 \mathrm{H}, \mathrm{CH}-\mathrm{O}), 6.69$ (d, $2 \mathrm{H}, \mathrm{Ar}), 6.79(\mathrm{t}, 1 \mathrm{H}, \mathrm{Ar}), 7.02(\mathrm{~s}, 1 \mathrm{H}, \mathrm{NH}=), 7.17(\mathrm{t}, 2 \mathrm{H}$, Ar), 7.38 (s, 1H, NH-Ph), $7.56(\mathrm{~s}, 1 \mathrm{H}, \mathrm{H}-3), 7.86(\mathrm{~s}, 1 \mathrm{H}$, $\mathrm{H}-6), 7.88$ (s, 1H, NH-NH-Ph), 9.07 (s, 1H, $\mathrm{NHSO}_{2}$ ) ppm; ${ }^{13} \mathrm{C}$ NMR (50 MHz, DMSO- $\left.d_{6}\right): \delta=19.22,36.44,65.06$, $102.35,112.99,120.01,128.16,129.09,130.56,132.04$, $136.00,136.64,140.28,148.23,159.25 \mathrm{ppm}$.

\section{1-[4-Chloro-5-methyl-2-[3-(trifluoromethyl)-} benzylthio]phenylsulfonyl]-3-(phenylamino)guanidine $\left(18, \mathrm{C}_{22} \mathrm{H}_{20} \mathrm{ClF}_{3} \mathrm{~N}_{4} \mathrm{O}_{2} \mathrm{~S}_{2}\right)$

Staring from $1.61 \mathrm{~g} 6$ and $0.50 \mathrm{~g}$ phenylhydrazine hydrochloride in $11 \mathrm{~cm}^{3}$ dry toluene for $1 \mathrm{~h}$, the title compound $\mathbf{1 8}$ was obtained. Yield: $1.26 \mathrm{~g}$ (68 \%); m.p.: $184-185{ }^{\circ} \mathrm{C}$; TLC: $R_{\mathrm{f}}=0.67\left(\mathrm{CHCl}_{3}:\right.$ pentane:acetone $\left.=1: 1: 0.5\right) ;$ IR $(\mathrm{KBr}): \bar{v}=3,444(\mathrm{NH}), 2,925\left(\mathrm{CH}_{3}, \mathrm{CH}_{2}\right), 1,330,1,120$ $\left(\mathrm{SO}_{2}\right) \mathrm{cm}^{-1} ;{ }^{1} \mathrm{H}$ NMR $\left(200 \mathrm{MHz}, \mathrm{DMSO}-d_{6}\right): \delta=2.30(\mathrm{~s}$, $\left.3 \mathrm{H}, \mathrm{CH}_{3}\right), 4.46$ (s, 2H, S- $\left.\mathrm{CH}_{2}\right), 6.66(\mathrm{~d}, 2 \mathrm{H}, \mathrm{Ar}), 6.77(\mathrm{t}, 1 \mathrm{H}$, Ar), 7.04 (s, 1H, NH=), 7.13 (t, 2H, Ar), 7.40 (s, 1H, NH-Ph), 7.47 (s, 1H, H-3), 7.55-7.68 (m, 2H, Ar), 7.74 (s, 1H, H-6), 7.97 (s, 1H, NH-NH-Ph), 7.88-7.97 (m, 2H, Ar), 9.08 (s, 1H, $\mathrm{NHSO}_{2}$ ) ppm; ${ }^{13} \mathrm{C}$ NMR (50 MHz, DMSO- $\left.d_{6}\right): \delta=19.22$, $35.90,112.94,119.97,124.20,124.27,126.01,126.08$, $128.42,129.05,129.89,130.77,132.49,133.44,134.90$, $136.59,138.39,140.47,148.16,159.19 \mathrm{ppm}$.

\section{1-[4-Chloro-5-methyl-2-[3-(trifluoromethyl)-}

benzylthio]phenylsulfonyl]-3-(4-methylphenylsulfonyl-

amino)guanidine $\left(19, \mathrm{C}_{23} \mathrm{H}_{22} \mathrm{ClF}_{3} \mathrm{~N}_{4} \mathrm{O}_{4} \mathrm{~S}_{3}\right)$

Starting from $1.61 \mathrm{~g} \mathrm{6}, 0.65 \mathrm{~g} p$-toluenesulfonyl hydrazide, and $0.66 \mathrm{~g}$ PTSA in $40 \mathrm{~cm}^{3}$ dry toluene for $1.5 \mathrm{~h}$, the title compound 19 was obtained. Yield: $1.49 \mathrm{~g}$ (70 \%); m.p.: 190-191 ${ }^{\circ} \mathrm{C}$; TLC: $R_{\mathrm{f}}=0.65\left(\mathrm{CHCl}_{3}: \mathrm{MeOH}=16: 3\right)$; IR $(\mathrm{KBr}): \bar{v}=3,459,3,360,3,310(\mathrm{NH}), 2,926\left(\mathrm{CH}_{3}, \mathrm{CH}_{2}\right)$, $1,635(\mathrm{C}=\mathrm{N}), 1,333,1,174,1,126\left(\mathrm{SO}_{2}\right) \mathrm{cm}^{-1} ;{ }^{1} \mathrm{H}$ NMR $\left(200 \mathrm{MHz}, \mathrm{DMSO}-d_{6}\right): \delta=2.29\left(\mathrm{~s}, 3 \mathrm{H}, \mathrm{CH}_{3}\right), 2.38(\mathrm{~s}, 3 \mathrm{H}$, $\left.\mathrm{CH}_{3}\right), 4.40$ (s, $\left.2 \mathrm{H}, \mathrm{S}-\mathrm{CH}_{2}\right), 7.24$ (brs, $\left.1 \mathrm{H}, \mathrm{NH}\right), 7.36$ (d, $2 \mathrm{H}$, Ar tosyl), $7.43(\mathrm{~s}, 1 \mathrm{H}, \mathrm{NH}), 7.50-7.77(\mathrm{~m}, 7 \mathrm{H}, \mathrm{Ar}, \mathrm{Ar}$ tosyl), $7.78(\mathrm{~s}, 1 \mathrm{H}, \mathrm{H}-6), 9.22\left(\mathrm{~s}, 1 \mathrm{H}, \mathrm{SO}_{2} \mathrm{NH}\right), 9.90(\mathrm{~s}, 1 \mathrm{H}$, $\left.\mathrm{SO}_{2} \mathrm{NH}\right) \mathrm{ppm} ;{ }^{13} \mathrm{C}$ NMR $\left(50 \mathrm{MHz}, \mathrm{DMSO}-d_{6}\right): \delta=19.22$, $21.32,36.01,124.31,124.39,126.00,126.08,128.13$, $128.89,129.51,129.89,130.59,132.68,133.46,134.69$, $134.73,136.69,138.41,140.23,144.18,158.46 \mathrm{ppm}$.

1-[4-Chloro-5-methyl-2-[4-(trifluoromethyl)benzylthio]phenylsulfonyl]-3-(phenylamino)guanidine $\left(\mathbf{2 0}, \mathrm{C}_{22} \mathrm{H}_{20} \mathrm{ClF}_{3} \mathrm{~N}_{4} \mathrm{O}_{2} \mathrm{~S}_{2}\right)$

Starting from $1.61 \mathrm{~g} 7$ and $0.51 \mathrm{~g}$ phenylhydrazine hydrochloride in $13 \mathrm{~cm}^{3}$ dry toluene for $2 \mathrm{~h}$, the title compound $\mathbf{2 0}$ was obtained. Yield: $1.04 \mathrm{~g}(56 \%)$; m.p.: $161-164{ }^{\circ} \mathrm{C}$; TLC: $R_{\mathrm{f}}=0.71\left(\mathrm{CHCl}_{3}:\right.$ pentane:acetone $\left.=1: 1: 0.5\right) ; \mathrm{IR}(\mathrm{KBr})$ : $\bar{v}=3,433(\mathrm{NH}), 2,924\left(\mathrm{CH}_{3}, \mathrm{CH}_{2}\right), 1,325,1,129\left(\mathrm{SO}_{2}\right)$ $\mathrm{cm}^{-1} ;{ }^{1} \mathrm{H}$ NMR $\left(500 \mathrm{MHz}\right.$, DMSO- $\left.d_{6}\right): \delta=2.29(\mathrm{~s}, 3 \mathrm{H}$, $\mathrm{CH}_{3}$ ), 4.46 (s, 2H, S- $\mathrm{CH}_{2}$ ), 6.65 (d, 2H, Ar), 6.76 (t, 1H, Ar), $7.04(\mathrm{~s}, 1 \mathrm{H}, \mathrm{NH}=), 7.11(\mathrm{t}, 2 \mathrm{H}, \mathrm{Ar}), 7.40(\mathrm{~s}, 1 \mathrm{H}, \mathrm{NH}-\mathrm{Ph}), 7.46$ (s, 1H, H-3), 7.62-7.72 (m, 4H, H-6, Ar, NH-NH-Ph), 7.89 (d, $2 \mathrm{H}, \mathrm{Ar}), 9.06\left(\mathrm{~s}, 1 \mathrm{H}, \mathrm{NHSO}_{2}\right) \mathrm{ppm} ;{ }^{13} \mathrm{C} \mathrm{NMR}(50 \mathrm{MHz}$, DMSO- $\left.d_{6}\right): \delta=19.21,35.81,112.94,119.96,125.48,125$. 56, 125.63, 128.03, 129.04, 130.12, 130.79, 132.39, 135.04, $136.65,140.29,141.74,148.15,159.21 \mathrm{ppm}$.

\section{1-[4-Chloro-5-methyl-2-(naphthalen-1-ylmethylthio)- phenylsulfonyl]-3-(phenylamino)guanidine}

\section{(21, $\mathrm{C}_{25} \mathrm{H}_{23} \mathrm{ClN}_{4} \mathrm{O}_{2} \mathrm{~S}_{2}$ )}

Starting from $1.54 \mathrm{~g} 8$ and $0.5 \mathrm{~g}$ phenylhydrazine hydrochloride in $10 \mathrm{~cm}^{3}$ dry toluene for $1 \mathrm{~h}$, the title compound 21 was obtained. Yield: $0.82 \mathrm{~g}(40 \%)$; m.p.: $145-150{ }^{\circ} \mathrm{C}$; TLC: $R_{\mathrm{f}}=$ $0.71\left(\mathrm{CHCl}_{3}\right.$ :pentane:acetone $\left.=1: 1: 0.5\right) ; \mathrm{IR}(\mathrm{KBr}): \bar{v}=3,331$ (NH), 2,922 $\left(\mathrm{CH}_{3}, \mathrm{CH}_{2}\right), 1,391,1,137\left(\mathrm{SO}_{2}\right) \mathrm{cm}^{-1} ;{ }^{1} \mathrm{H} \mathrm{NMR}$ $\left(200 \mathrm{MHz}, \mathrm{DMSO}-d_{6}\right): \delta=2.33\left(\mathrm{~s}, 3 \mathrm{H}, \mathrm{CH}_{3}\right), 4.79(\mathrm{~s}, 2 \mathrm{H}$, $\left.\mathrm{S}-\mathrm{CH}_{2}\right), 6.62(\mathrm{~d}, 2 \mathrm{H}, \mathrm{Ar}), 6.75(\mathrm{t}, 1 \mathrm{H}, \mathrm{Ar}), 6.96(\mathrm{~s}, 1 \mathrm{H}, \mathrm{NH}=)$, 7.07-7.10 (m, 2H, Ar), 7.33 (s, 1H, NH-Ph), 7.43-7.46 (m, 1H, Ar), 7.52-7.61 (m, 4H, H-3, Ar), 7.84-7.96 (m, 4H, H-6, Ar, $\mathrm{NH}$-NH-Ph), 8.25 (d, 1H, Ar), 9.05 (s, 1H, $\mathrm{NHSO}_{2}$ ) ppm; ${ }^{13} \mathrm{C}$ NMR (50 MHz, DMSO- $\left.d_{6}\right): \delta=19.28,34.82,112.92,119$. $97,124.50,125.82,126.24,126.60,128.22,128.41,128.80,12$ $8.89,129.06,130.73,131.71,132.00,132.24,133.69,136.19$, 136.72, 140.13, 148.13, $159.19 \mathrm{ppm}$.

\section{1-[4-Chloro-5-methyl-2-(naphthalen-1-ylmethylthio)- phenylsulfonyl]-3-(4-chlorophenylamino)guanidine $\left(\mathbf{2 2}, \mathrm{C}_{25} \mathrm{H}_{22} \mathrm{Cl}_{2} \mathrm{~N}_{4} \mathrm{O}_{2} \mathrm{~S}_{2}\right)$}

Starting from $1.54 \mathrm{~g} 8$ and $0.63 \mathrm{~g}$ 4-chlorophenylhydrazine hydrochloride in $10 \mathrm{~cm}^{3}$ dry toluene for $3 \mathrm{~h}$, the title compound 22 was obtained. Yield: $1.34 \mathrm{~g}$ (70 \%); m.p.: 148 $-149{ }^{\circ} \mathrm{C}$; TLC: $R_{\mathrm{f}}=0.68\left(\mathrm{CHCl}_{3}:\right.$ pentane:acetone $=1: 1$ : 0.5); IR (KBr): $\bar{v}=3,448,3,318(\mathrm{NH}), 2,923\left(\mathrm{CH}_{3}, \mathrm{CH}_{2}\right)$, $1,340,1,140\left(\mathrm{SO}_{2}\right) \mathrm{cm}^{-1} ;{ }^{1} \mathrm{H}$ NMR $\left(200 \mathrm{MHz}, \mathrm{DMSO}-d_{6}\right)$ : $\delta=2.33\left(\mathrm{~s}, 3 \mathrm{H}, \mathrm{CH}_{3}\right), 4.80\left(\mathrm{~s}, 2 \mathrm{H}, \mathrm{S}-\mathrm{CH}_{2}\right), 6.60(\mathrm{~d}, 2 \mathrm{H}, \mathrm{Ar})$, $6.98(\mathrm{~s}, 1 \mathrm{H}, \mathrm{NH}=), 7.07$ (d, 2H, Ar), 7.35-7.64 (m, 6H, Ar, $\mathrm{NH}), 7.84-8.10(\mathrm{~m}, 4 \mathrm{H}, \mathrm{Ar}, \mathrm{NH}), 8.50$ (d, 1H, Ar), 9.05 (s, $\left.1 \mathrm{H}, \mathrm{NHSO}_{2}\right) \mathrm{ppm} ;{ }^{13} \mathrm{C}$ NMR $\left(50 \mathrm{MHz}\right.$, DMSO- $\left.d_{6}\right): \delta=$ 19.27, 34.77, 114.35, 123.23, 124.51, 125.81, 126.25, 126. $60,128.27,128.45,128.78,130.72,131.73,131.98,132.22$, 133.68, 136.22, 136.77, 139.97, 147.18, $159.01 \mathrm{ppm}$.

\section{1-[4-Chloro-5-methyl-2-(naphthalen-1-ylmethylthio)-} phenylsulfonyl]-3-(4-methylphenylsulfonylamino)guanidine (23, $\mathrm{C}_{26} \mathrm{H}_{25} \mathrm{ClN}_{4} \mathrm{O}_{4} \mathrm{~S}_{3}$ )

Starting from $1.54 \mathrm{~g} \mathrm{8}, 0.65 \mathrm{~g} p$-toluenesulfonyl hydrazide, and $0.66 \mathrm{~g}$ PTSA in $70 \mathrm{~cm}^{3}$ dry toluene for $2.5 \mathrm{~h}$, the title compound 23 was obtained. Yield: $1.61 \mathrm{~g}$ (78 \%); m.p.: 203-206 ${ }^{\circ} \mathrm{C}$; TLC: $\quad R_{\mathrm{f}}=0.32 \quad\left(\mathrm{CHCl}_{3}:\right.$ pentane:acetone 
$=1: 1: 0.5) ; \mathrm{IR}(\mathrm{KBr}): \bar{v}=3,475,3,370,3,310(\mathrm{NH}), 2,923$ $\left(\mathrm{CH}_{3}, \mathrm{CH}_{2}\right), 1633(\mathrm{C}=\mathrm{N}), 1,339,1,172,1,146\left(\mathrm{SO}_{2}\right) \mathrm{cm}^{-1}$; ${ }^{1} \mathrm{H}$ NMR (200 MHz, DMSO-d $\left.d_{6}\right): \delta=2.33\left(\mathrm{~s}, 3 \mathrm{H}, \mathrm{CH}_{3}\right), 2$. 36 (s, 3H, $\mathrm{CH}_{3}$ ), 4.74 (s, 2H, S- $\mathrm{CH}_{2}$ ), 7.20 (brs, 1H, NH), 7.31 (d, 2H, Ar tosyl), 7.44 (s, 1H, NH), 7.48-7.74 (m, 6H, Ar naphth, tosyl), 7.66 (s, 1H, H-3), 7.82 (s, 1H, H-6), 7.8 4-8.02 (m, 2H, Ar naphth), 8.24 (d, 1H, Ar naphth), 9.20 (s, $\left.1 \mathrm{H}, \mathrm{SO}_{2} \mathrm{NH}\right), 9.86\left(\mathrm{~s}, 1 \mathrm{H}, \mathrm{SO}_{2} \mathrm{NH}\right) \mathrm{ppm} ;{ }^{13} \mathrm{C} \mathrm{NMR}$ $\left(50 \mathrm{MHz}, \mathrm{DMSO}-d_{6}\right): \delta=19.28,21.33,34.97,124.49,12$ 5.79, 126.24, 126.58, 128.12, 128.22, 128.41, 128.80, 128. $96,129.86,130.57,131.67,132.07,132.46,133.68,134.6$ 5, 135.97, 136.79, 139.94, 144.13, 158.45 ppm.

1-[4-Chloro-2-(1,2-dihydro-2-oxoquinolin-4-ylmethylthio)5-methylphenylsulfonyl]-3-(phenylamino)guanidine

(24, $\mathrm{C}_{24} \mathrm{H}_{22} \mathrm{ClN}_{5} \mathrm{O}_{3} \mathrm{~S}_{2}$ )

Starting from $1.6 \mathrm{~g} 9$ and $0.51 \mathrm{~g}$ phenylhydrazine hydrochloride in $15 \mathrm{~cm}^{3}$ dry toluene for $8 \mathrm{~h}$, the title compound 24 was obtained. Yield: $1.06 \mathrm{~g}$ (58 \%); m.p.: $171-173{ }^{\circ} \mathrm{C}$; TLC: $R_{\mathrm{f}}=0.70\left(\mathrm{CHCl}_{3}: \mathrm{MeOH}=16: 3\right)$; IR $(\mathrm{KBr}): \bar{v}=$ 3,343 (NH), 2,922 $\left(\mathrm{CH}_{3}, \mathrm{CH}_{2}\right), 1,663(\mathrm{CO}), 1,386,1,143$ $\left(\mathrm{SO}_{2}\right) \mathrm{cm}^{-1} ;{ }^{1} \mathrm{H}$ NMR $\left(500 \mathrm{MHz}, \mathrm{DMSO}-d_{6}\right): \delta=2.31(\mathrm{~s}$, $\left.3 \mathrm{H}, \mathrm{CH}_{3}\right), 4.62\left(\mathrm{~s}, 2 \mathrm{H}, \mathrm{S}-\mathrm{CH}_{2}\right), 6.66(\mathrm{~d}, 2 \mathrm{H}, \mathrm{Ar}), 6.69$ $(\mathrm{s}, 1 \mathrm{H}, \mathrm{Ar}), 6.76(\mathrm{t}, 1 \mathrm{H}, \mathrm{Ar}), 7.00(\mathrm{~s}, 1 \mathrm{H}, \mathrm{NH}=), 7.13$ (t, 2H, Ar), 7.22 (t, 1H, Ar), 7.32 (d, 1H, Ar), 7.39 (s, 1H, NH-Ph), 7.50 (t, 1H, Ar), 7.52 (s, 1H, H-3), 7.88 (s, 1H, H-6), 7.90 (s, 1H, NH-NH-Ph), 7.94 (d, 1H, Ar), 9.14 (s, $1 \mathrm{H}, \mathrm{NHSO}_{2}$ ), 11.78 (s, 1H, NH-quinol) ppm; ${ }^{13} \mathrm{C} \mathrm{NMR}$ (50 MHz, DMSO- $\left.d_{6}\right): \delta=19.03,33.16,112.69,115.69$, $118.31,119.75,121.67,121.83,125.01,128.46,128.85,13$ $0.51,130.61,132.64,134.39,136.49,138.90,140.48,14$ $6.08,147.88,158.91,161.55 \mathrm{ppm}$.

\section{1-[4-Chloro-2-(2,3-dihydrobenzo[b][1,4]dioxin-2-}

ylmethylthio)-5-methylphenylsulfonyl]-3-(phenylamino)-

guanidine $\left(\mathbf{2 5}, \mathrm{C}_{23} \mathrm{H}_{23} \mathrm{ClN}_{4} \mathrm{O}_{4} \mathrm{~S}_{2}\right)$

Starting from $1.57 \mathrm{~g} \mathbf{1 0}$ and $0.53 \mathrm{~g}$ phenylhydrazine hydrochloride in $8 \mathrm{~cm}^{3}$ dry toluene for $1 \mathrm{~h}$, the title compound 25 was obtained. Yield: 1.12 g (62 \%); m.p.: 175-177 ${ }^{\circ} \mathrm{C}$; TLC: $R_{\mathrm{f}}=0.70\left(\mathrm{CHCl}_{3}\right.$ :pentane:acetone $=$ 1:1:0.5); IR (KBr): $\bar{v}=3,442(\mathrm{NH}), 2,923\left(\mathrm{CH}_{3}, \mathrm{CH}_{2}\right)$, $1,399,1,145\left(\mathrm{SO}_{2}\right) \mathrm{cm}^{-1} ;{ }^{1} \mathrm{H}$ NMR (500 MHz, DMSO- $\left.d_{6}\right)$ : $\delta=2.33\left(\mathrm{~s}, 3 \mathrm{H}, \mathrm{CH}_{3}\right), 3.32-3.48\left(\mathrm{~m}, 2 \mathrm{H}, \mathrm{S}-\mathrm{CH}_{2}\right), 4.06$ -4.10 (m, 1H, CH-O), 4.37 (d, 2H, $\left.\mathrm{CH}_{2}-\mathrm{O}\right), 6.68$ (d, 2H, Ar), 6.77 (t, 1H, Ar), 6.81-6.87 (m, 4H, Ar), 7.03 (s, 1H, N $\mathrm{H}=), 7.15$ (t, 2H, Ar), 7.41 (s, 1H, NH-Ph), 7.64 (s, 1H, H-3), 7.88 (s, 1H, H-6), 7.90 (s, 1H, NH-NH-Ph), 9.09 (s, $\left.1 \mathrm{H}, \mathrm{NHSO}_{2}\right) \mathrm{ppm} ;{ }^{13} \mathrm{C} \mathrm{NMR}\left(50 \mathrm{MHz}, \mathrm{DMSO}-d_{6}\right): \delta=$ 19.29, 33.19, 66.32, 72.02, 112.96, 117.23, 117.41, 120.03, $121.66,121.83,128.87,129.11,130.74,132.78,134.89$, $136.86,140.95,142.77,143.10,148.19,159.23$ ppm.
General procedure for the preparation of 4-chloro-5methyl-2-( $R^{1}$-methylthio $)-N-\left(1-R^{2}-4,5-\right.$ dihydro-5-oxo$1 H$-1,2,4-triazol-3-yl)benzenesulfonamide derivatives 26-40

The reaction was carried out in a two-neck round-bottom flask (capacity $5 \mathrm{~cm}^{3}$ ) with drying tube protection. To the cooled $\left(0{ }^{\circ} \mathrm{C}\right)$ mixture of the corresponding aminoguanidines 11-25 (1 mmol) in dry THF, $0.46 \mathrm{~cm}^{3}$ TsNCO ( $3 \mathrm{mmol}$ ) was added dropwise, and the reaction mixture was stirred at room temperature for $1 \mathrm{~h}$, then at reflux for 8-36 h. After cooling $\left(0{ }^{\circ} \mathrm{C}\right.$, overnight $)$ the reaction product was isolated in precipitate state $(\mathbf{2 7 - 3 1}, \mathbf{3 4 - 4 0})$ or in oil form $(26,32$, and 33) and purified by crystallization from ethanol (26-31, 33-39), ethyl acetate (32), or acetonitrile (40).

2-(Benzylthio)-4-chloro-N-(4,5-dihydro-5-oxo-1H-1,2,4triazol-3-yl)-5-methylbenzenesulfonamide

(26, $\mathrm{C}_{16} \mathrm{H}_{15} \mathrm{ClN}_{4} \mathrm{O}_{3} \mathrm{~S}_{2}$ )

Starting from $0.385 \mathrm{~g} 11$ (1 mmol) in $1.5 \mathrm{~cm}^{3} \mathrm{THF}$, the reaction mixture was refluxed for $8 \mathrm{~h}$. After cooling to room temperature, the oily solution was treated with $30 \mathrm{~cm}^{3}$ diethyl ether. The ether solution was decanted from the solid, evaporated to dryness, and the residue crystallized from ethanol to obtain $0.065 \mathrm{~g}$ (16\%) of $\mathbf{2 6}$. The deposit after decantation was treated with $20 \mathrm{~cm}^{3}$ diethyl ether, filtered off, and purified by crystallization from ethanol to give $0.123 \mathrm{~g}(30 \%)$ as a second fraction of 26. M.p.: $278-279{ }^{\circ} \mathrm{C}$; TLC: $R_{\mathrm{f}}=0.44$ (benzene:EtOH = 2:1); IR (KBr): $\bar{v}=3,346(\mathrm{NH}), 2,929$ $\left(\mathrm{CH}_{3}, \mathrm{CH}_{2}\right), 1,688(\mathrm{CO}), 1,355,1,161\left(\mathrm{SO}_{2}\right) \mathrm{cm}^{-1} ;{ }^{1} \mathrm{H} \mathrm{NMR}$ $\left(500 \mathrm{MHz}, \mathrm{DMSO}-d_{6}\right): \delta=2.32\left(\mathrm{~s}, 3 \mathrm{H}, \mathrm{CH}_{3}\right), 4.36(\mathrm{~s}, 2 \mathrm{H}$, $\mathrm{S}-\mathrm{CH}_{2}$ ), 7.26 (t, 1H, Ar), 7.32 (t, 2H, Ar), 7.43 (d, 2H, Ar), 7.54 (s, 1H, H-3), 7.86 (s, 1H, H-6), 11.19 (s, 1H, NH), 11.50 (s, 1H, NH) ppm; ${ }^{13} \mathrm{C}$ NMR (50 MHz, DMSO- $\left.d_{6}\right): \delta=19.29,36.81$, 125.89, 127.63, 128.73, 129.49, 132.02, 132.78, 136.05, 136.29, 138.29, 154.50 ppm; LC-MS (IT-TOF): $\mathrm{m} / z=410$ $\left(\mathrm{M}^{+}\right), t_{\mathrm{R}}=5 \mathrm{~min}$.

2-(Benzylthio)-4-chloro-N-(4,5-dihydro-1-methyl-5oxo-1H-1,2,4-triazol-3-yl)-5-methylbenzenesulfonamide (27, $\left.\mathrm{C}_{17} \mathrm{H}_{17} \mathrm{ClN}_{4} \mathrm{O}_{3} \mathrm{~S}_{2}\right)$

Starting from $0.399 \mathrm{~g} 12$ (1 mmol) in $1.5 \mathrm{~cm}^{3}$ THF, the reaction mixture was refluxed for $8 \mathrm{~h}$. The product was purified to give $0.263 \mathrm{~g}(62 \%)$ of 27. M.p.: $226-228{ }^{\circ} \mathrm{C}$; TLC: $R_{\mathrm{f}}=0.22$ (benzene:EtOH $\left.=2: 1\right)$; IR $(\mathrm{KBr}): \bar{v}=3$, $102(\mathrm{NH}), 2,924\left(\mathrm{CH}_{3}, \mathrm{CH}_{2}\right), 1,764(\mathrm{CO}), 1,319,1,131$ $\left(\mathrm{SO}_{2}\right) \mathrm{cm}^{-1} ;{ }^{1} \mathrm{H} \mathrm{NMR}\left(500 \mathrm{MHz}, \mathrm{DMSO}-d_{6}\right): \delta=2.31(\mathrm{~s}$, $\left.3 \mathrm{H}, \mathrm{CH}_{3}\right), 3.19$ (s, 3H, $\left.\mathrm{CH}_{3}\right), 4.32\left(\mathrm{~s}, 2 \mathrm{H}, \mathrm{S}-\mathrm{CH}_{2}\right), 7.25$ (t, $1 \mathrm{H}$, Ar), 7.31 (t, 2H, Ar), 7.37 (d, 2H, Ar), 7.51 (s, 1H, H-3), 7.94 (s, 1H, H-6), 11.75 (brs, 1H, NH) ppm; ${ }^{13} \mathrm{C}$ NMR $(50 \mathrm{MHz}$, DMSO- $\left.d_{6}\right): \delta=19.21,32.66,36.38,127.51,128.04,128.71$, $129.20,130.93,132.18,135.60,136.67,136.84,139.58$, 
147.70, 152.49 ppm; LC-MS (IT-TOF): $m / z=424\left(\mathrm{M}^{+}\right)$, $t_{\mathrm{R}}=6 \mathrm{~min}$.

2-(Benzylthio)-4-chloro-N-(4,5-dihydro-5-oxo-1-phenyl1H-1,2,4-triazol-3-yl)-5-methylbenzenesulfonamide

$\left(28, \mathrm{C}_{22} \mathrm{H}_{19} \mathrm{ClN}_{4} \mathrm{O}_{3} \mathrm{~S}_{2}\right)$

Starting from $0.461 \mathrm{~g} 13(1 \mathrm{mmol})$ in $1.5 \mathrm{~cm}^{3} \mathrm{THF}$, the reaction mixture was refluxed for $9 \mathrm{~h}$. The product was purified to give $0.362 \mathrm{~g}(74 \%)$ of $\mathbf{2 8}$. M.p.: $212-214.5^{\circ} \mathrm{C}$; TLC: $R_{\mathrm{f}}=0.61$ (benzene:EtOH =2:1); IR $(\mathrm{KBr}): \bar{v}=$ 3,240 (NH), 2,923 $\left(\mathrm{CH}_{3}, \mathrm{CH}_{2}\right), 1,702(\mathrm{CO}), 1,354,1,173$ $\left(\mathrm{SO}_{2}\right) \mathrm{cm}^{-1} ;{ }^{1} \mathrm{H}$ NMR $\left(200 \mathrm{MHz}, \mathrm{DMSO}-d_{6}\right): \delta=2.36(\mathrm{~s}$, $\left.3 \mathrm{H}, \mathrm{CH}_{3}\right), 4.34$ (s, 2H, S-CH $\left.{ }_{2}\right), 7.12-7.24$ (m, 4H, Ar), 7. 30-7.44 (m, 4H, Ar), 7.58 (s, 1H, H-3), 7.66 (d, 2H, Ar), 7.98 (s, 1H, H-6), 11.98 (s, 1H, NH) ppm; ${ }^{13} \mathrm{C}$ NMR $(50 \mathrm{MHz}$, DMSO- $\left.d_{6}\right): \delta=19.21,36.70,117.66,124.74,127.60,128$. 49, 128.63, 129.19, 129.44, 132.80, 133.21, 135.55, 135.85, $136.71,137.91,138.92,139.52,151.52 \mathrm{ppm}$; LC-MS (IT-TOF): $\mathrm{m} / \mathrm{z}=486\left(\mathrm{M}^{+}\right), t_{\mathrm{R}}=13 \mathrm{~min}$.

\section{2-(Benzylthio)-4-chloro- $N$-[4,5-dihydro-1-}

(4-nitrophenylsulfonyl)-5-oxo-1H-1,2,4-triazol-3-yl]-5-

methylbenzenesulfonamide $\left(\mathbf{2 9}, \mathrm{C}_{22} \mathrm{H}_{18} \mathrm{ClN}_{5} \mathrm{O}_{7} \mathrm{~S}_{3}\right)$

Starting from $0.596 \mathrm{~g} 14(1 \mathrm{mmol})$ in $2 \mathrm{~cm}^{3}$ THF, the reaction mixture was refluxed for $9 \mathrm{~h}$. The product was purified to give $0.30 \mathrm{~g}(50 \%)$ of 29. M.p.: $211-214{ }^{\circ} \mathrm{C}$; TLC: $R_{\mathrm{f}}=0.59$ (benzene:ethanol $=2: 1$ ); IR $(\mathrm{KBr}): \bar{v}=3$, 429, 3,269 (NH), 1,764 (CO), 1,536, 1,350 $\left(\mathrm{NO}_{2}\right), 1,403$, 1,391, 1,184, 1,167 $\left(\mathrm{SO}_{2}\right) \mathrm{cm}^{-1} ;{ }^{1} \mathrm{H}$ NMR $(200 \mathrm{MHz}$, DMSO- $\left.d_{6}\right): \delta=2.32\left(\mathrm{~s}, 3 \mathrm{H}, \mathrm{CH}_{3}\right), 4.28\left(\mathrm{~s}, 2 \mathrm{H}, \mathrm{S}-\mathrm{CH}_{2}\right), 7$. 10-7.21 (m, 3H, Ar), 7.30 (d, 2H, Ar), 7.50 (s, 1H, H-3), 7.78 (d, $2 \mathrm{H}, J=8.8 \mathrm{~Hz}, \mathrm{Ar}), 7.89$ (s, 1H, H-6), 8.24 (d, $2 \mathrm{H}$, $J=8.8 \mathrm{~Hz}, \mathrm{Ar}) \mathrm{ppm} ;{ }^{13} \mathrm{C} \mathrm{NMR}\left(50 \mathrm{MHz}, \mathrm{DMSO}-d_{6}\right)$ : $\delta=19.19,36.41,124.92,127.42,128.30,128.40,128.47$, $128.82,129.16,132.48,133.47,135.98,136.15,138.37$, 141.76, 144.95, 150.91, 151.71 ppm; LC-MS (IT-TOF): $\mathrm{m} / \mathrm{z}=596\left(\mathrm{M}^{+}\right), t_{\mathrm{R}}=15 \mathrm{~min}$.

2-(Benzylthio)-4-chloro-N-[4,5-dihydro-1-(4-methylphenylsulfonyl)-5-oxo-1H-1,2,4-triazol-3-yl]-5-methylbenzenesulfonamide (30, $\left.\mathrm{C}_{23} \mathrm{H}_{21} \mathrm{ClN}_{4} \mathrm{O}_{5} \mathrm{~S}_{3}\right)$

Starting from $0.539 \mathrm{~g} 15(1 \mathrm{mmol})$ in $1.5 \mathrm{~cm}^{3} \mathrm{THF}$, the reaction mixture was refluxed for $8 \mathrm{~h}$. The product was purified to give $0.405 \mathrm{~g}$ (72\%) of 30. M.p.: $202-204{ }^{\circ} \mathrm{C}$; TLC: $R_{\mathrm{f}}=0.60$ (benzene:EtOH $\left.=2: 1\right)$; IR $(\mathrm{KBr}): \bar{v}=3,3$ $71(\mathrm{NH}), 2,922\left(\mathrm{CH}_{3}, \mathrm{CH}_{2}\right), 1,755(\mathrm{CO}), 1,387,1,191,1,176$ $\left(\mathrm{SO}_{2}\right) \mathrm{cm}^{-1} ;{ }^{1} \mathrm{H}$ NMR $\left(500 \mathrm{MHz}, \mathrm{DMSO}-d_{6}\right): \delta=2.34$ (s, $\left.3 \mathrm{H}, \mathrm{CH}_{3}\right), 2.37$ (s, $\left.3 \mathrm{H}, \mathrm{CH}_{3}\right), 4.28$ (s, $\left.2 \mathrm{H}, \mathrm{S}-\mathrm{CH}_{2}\right), 7.20$ (t, 1H, Ar), $7.26(\mathrm{~d}, 2 \mathrm{H}, \mathrm{Ar}), 7.33(\mathrm{~d}, 2 \mathrm{H}, \mathrm{Ar}), 7.36(\mathrm{~d}, 2 \mathrm{H}$, Ar), 7.51 (d, 2H, Ar), 7.54 (s, 1H, H-3), 7.89 (s, 1H, H-6), $11.93(\mathrm{~s}, 1 \mathrm{H}, \mathrm{NH}) \mathrm{ppm} ;{ }^{13} \mathrm{C}$ NMR $\left(50 \mathrm{MHz}, \mathrm{DMSO}-d_{6}\right)$ : $\delta=19.23,21.46,36.67,127.32,127.60,128.52,128.66,12$ $9.32,130.18,132.69,133.33,133.95,135.42,135.86,136.4$
2, 138.77, 143.34, 145.82, 151.67 ppm; LC-MS (IT-TOF): $\mathrm{m} / \mathrm{z}=564\left(\mathrm{M}^{+}\right), t_{\mathrm{R}}=12 \mathrm{~min}$.

4-Chloro-N-[4,5-dihydro-1-(4-methylphenyl)-5-oxo-1H1,2,4-triazol-3-yl]-2-(ethoxycarbonylmethylthio)-5-methylbenzenesulfonamide $\left(\mathbf{3 1}, \mathrm{C}_{20} \mathrm{H}_{21} \mathrm{ClN}_{4} \mathrm{O}_{5} \mathrm{~S}_{2}\right)$

Starting from $0.471 \mathrm{~g} 16(1 \mathrm{mmol})$ in $1.5 \mathrm{~cm}^{3}$ THF, the reaction mixture was refluxed for $8 \mathrm{~h}$. The precipitate of byproducts was filtered off. The filtrate was evaporated to dryness under reduced pressure and purified to give $0.343 \mathrm{~g}$ $(69 \%)$ of 31. M.p.: $190-191{ }^{\circ} \mathrm{C}$; TLC: $R_{\mathrm{f}}=0.42$ (benzene:EtOH = 2:1); IR (KBr): $\bar{v}=3,255(\mathrm{NH}), 2,978,2,801$ $\left(\mathrm{CH}_{3}, \mathrm{CH}_{2}\right), 1,726(\mathrm{CO}), 1,336,1,171\left(\mathrm{SO}_{2}\right) \mathrm{cm}^{-1} ;{ }^{1} \mathrm{H} \mathrm{NMR}$ $\left(200 \mathrm{MHz}, \mathrm{DMSO}-d_{6}\right): \delta=1.04\left(\mathrm{t}, 3 \mathrm{H}, \mathrm{CH}_{3}\right), 2.26(\mathrm{~s}, 3 \mathrm{H}$, $\mathrm{CH}_{3}$ ), 2.38 (s, $\left.3 \mathrm{H}, \mathrm{CH}_{3}\right), 3.97-4.08\left(\mathrm{~m}, 4 \mathrm{H}, \mathrm{S}-\mathrm{CH}_{2}, \mathrm{CH}_{2}\right.$ ), 7.18 (d, 2H, Ar), 7.52 (d, 2H, Ar), 7.60 (s, 1H, H-3), 8.01 (s, $1 \mathrm{H}, \mathrm{H}-6), 11.98(\mathrm{~s}, 1 \mathrm{H}, \mathrm{NH}) \mathrm{ppm} ;{ }^{13} \mathrm{C}$ NMR $(50 \mathrm{MHz}$, DMSO- $\left.d_{6}\right): \delta=14.10,19.23,20.66,35.15,61.43,117.76$, $129.14,129.54,133.20,133.51,133.90,135.33,135.51$, $136.11,138.90,139.23,151.40,168.89$ ppm; LC-MS (IT-TOF): $m / z=496\left(\mathrm{M}^{+}\right), t_{\mathrm{R}}=12 \mathrm{~min}$.

4-Chloro-N-(4,5-dihydro-5-oxo-1-phenyl-1H-1,2,4triazol-3-yl)-2-(1,3-dioxolan-2-ylmethylthio)-5methylbenzenesulfonamide $\left(\mathbf{3 2}, \mathrm{C}_{19} \mathrm{H}_{19} \mathrm{ClN}_{4} \mathrm{O}_{5} \mathrm{~S}_{2}\right)$ Starting from $0.458 \mathrm{~g} 17(1 \mathrm{mmol})$ in $3 \mathrm{~cm}^{3}$ THF, the reaction mixture was refluxed for $5 \mathrm{~h}$. After cooling to room temperature, the oily residue was treated with diethyl ether to obtain a white solid. The crude product was purified to give $0.159 \mathrm{~g}(33 \%)$ of 32. M.p.: $214-217{ }^{\circ} \mathrm{C}$; TLC: $R_{\mathrm{f}}=0.36$ (benzene:EtOH = 2:1); IR (KBr): $\bar{v}=3,414(\mathrm{NH}), 2,972$ $\left(\mathrm{CH}_{3}, \mathrm{CH}_{2}\right), 1,716(\mathrm{CO}), 1,382,1,165\left(\mathrm{SO}_{2}\right) \mathrm{cm}^{-1} ;{ }^{1} \mathrm{H} \mathrm{NMR}$ $\left(500 \mathrm{MHz}, \mathrm{DMSO}-d_{6}\right): \delta=2.37\left(\mathrm{~s}, 3 \mathrm{H}, \mathrm{CH}_{3}\right), 3.28(\mathrm{~d}, 2 \mathrm{H}$, $\left.\mathrm{S}-\mathrm{CH}_{2}\right), 3.71-3.75\left(\mathrm{~m}, 2 \mathrm{H}, \mathrm{CH}_{2}-\mathrm{O}\right), 3.83-3.88(\mathrm{~m}, 2 \mathrm{H}$, $\left.\mathrm{CH}_{2}-\mathrm{O}\right), 5.05(\mathrm{t}, 1 \mathrm{H}, \mathrm{CH}-\mathrm{O}), 7.13(\mathrm{t}, 1 \mathrm{H}, \mathrm{Ar}), 7.36(\mathrm{t}, 2 \mathrm{H}$, $\mathrm{Ar}), 7.64$ (d, 2H, Ar), 7.68 (s, 1H, H-3), 7.97 (s, 1H, H-6), $11.97(\mathrm{~s}, 1 \mathrm{H}, \mathrm{NH}) \mathrm{ppm} ;{ }^{13} \mathrm{C}$ NMR $\left(50 \mathrm{MHz}, \mathrm{DMSO}-d_{6}\right)$ : $\delta=19.22,36.70,64.96,102.10,117.69,124.73,129.18$, $129.31,129.83,132.98,136.13,136.74,137.89,138.87$, 139.64, $151.54 \mathrm{ppm}$; LC-MS (IT-TOF): $m / z=482\left(\mathrm{M}^{+}\right)$, $t_{\mathrm{R}}=9 \mathrm{~min}$.

4-Chloro-N-(4,5-dihydro-5-oxo-1-phenyl-1H-1,2,4triazol-3-yl)-5-methyl-2-[3-(trifluoromethyl)benzylthio]benzenesulfonamide $\left(\mathbf{3 3}, \mathrm{C}_{23} \mathrm{H}_{18} \mathrm{ClF}_{3} \mathrm{~N}_{4} \mathrm{O}_{3} \mathrm{~S}_{2}\right)$ Starting from $0.506 \mathrm{~g} 18(1 \mathrm{mmol})$ in $1 \mathrm{~cm}^{3}$ THF, the reaction mixture was refluxed for $9 \mathrm{~h}$. After cooling to room temperature, the oily residue was treated with diethyl ether to obtain a white solid. The crude product was purified to give $0.210 \mathrm{~g}(38 \%)$ of 33. M.p.: $195-198{ }^{\circ} \mathrm{C}$; TLC: $R_{\mathrm{f}}=0.45$ (benzene:EtOH = 2:1); IR (KBr): $\bar{v}=3,425(\mathrm{NH}), 2,924$ $\left(\mathrm{CH}_{3}, \mathrm{CH}_{2}\right), 1,702(\mathrm{CO}), 1,334,1,170\left(\mathrm{SO}_{2}\right) \mathrm{cm}^{-1} ;{ }^{1} \mathrm{H} \mathrm{NMR}$ $\left(200 \mathrm{MHz}, \mathrm{DMSO}-d_{6}\right): \delta=2.36\left(\mathrm{~s}, 3 \mathrm{H}, \mathrm{CH}_{3}\right), 4.48(\mathrm{~s}, 2 \mathrm{H}$, 
S-CH $\left.\mathrm{CH}_{2}\right), 7.14(\mathrm{t}, 1 \mathrm{H}, \mathrm{Ar}), 7.27-7.58(\mathrm{~m}, 4 \mathrm{H}, \mathrm{Ar}), 7.58(\mathrm{~s}, 1 \mathrm{H}$, H-3), 7.62-7.76 (m, 4H, Ar), 7.98 (s, 1H, H-6), 12.00 (s, 1H, $\mathrm{NH}) \mathrm{ppm} ;{ }^{13} \mathrm{C}$ NMR $\left(50 \mathrm{MHz}\right.$, DMSO- $\left.d_{6}\right): \delta=19.20$, $36.05,117.62$, 124.20, 124.29, 124.71, 126.08, 126.16, $128.93,129.14,129.32,129.56,133.24,133.33,133.43$, 135.44, 136.33, 137.85, 138.81, 139.48, 151.49 ppm; LC-MS (IT-TOF): $m / z=554\left(\mathrm{M}^{+}\right), t_{\mathrm{R}}=17 \mathrm{~min}$.

\section{4-Chloro-N-[4,5-dihydro-1-(4-methylphenylsulfonyl)- 5-oxo-1H-1,2,4-triazol-3-yl]-5-methyl-2-[3-(trifluoro- methyl)benzylthio]benzenesulfonamide}

$\left(34, \mathrm{C}_{24} \mathrm{H}_{20} \mathrm{ClF}_{3} \mathrm{~N}_{4} \mathrm{O}_{5} \mathrm{~S}_{3}\right)$

Starting from $0.607 \mathrm{~g} 19(1 \mathrm{mmol})$ in $2 \mathrm{~cm}^{3}$ THF, the reaction mixture was refluxed for $36 \mathrm{~h}$. The product was purified to give $0.443 \mathrm{~g}(71 \%)$ of 34. M.p.: $99-100{ }^{\circ} \mathrm{C}$; TLC: $R_{\mathrm{f}}=0.62$ (benzene:EtOH = 2:1); IR $(\mathrm{KBr}): \bar{v}=3,284(\mathrm{NH}), 2,924\left(\mathrm{CH}_{3}\right.$, $\left.\mathrm{CH}_{2}\right), 1,716$ (CO), 1,331, 1,347, 1,170, $1,194\left(\mathrm{SO}_{2}\right) \mathrm{cm}^{-1} ;{ }^{1} \mathrm{H}$ NMR (500 MHz, DMSO- $\left.d_{6}\right): \delta=2.32\left(\mathrm{~s}, 3 \mathrm{H}, \mathrm{CH}_{3}\right), 2.34$ (s, $\left.3 \mathrm{H}, \mathrm{CH}_{3}\right), 4.38$ (s, $\left.2 \mathrm{H}, \mathrm{S}-\mathrm{CH}_{2}\right), 7.30$ (d, 2H, Ar), 7.43-7.52 (m, 4H, Ar), 7.55-7.69 (m, 3H, Ar), 7.86 (s, 1H, H-6), 11.90 (brs, $1 \mathrm{H}$, $\mathrm{NH}) \mathrm{ppm} ;{ }^{13} \mathrm{C}$ NMR $\left(50 \mathrm{MHz}\right.$, DMSO- $\left.d_{6}\right): \delta=19.24,21.36$, $36.03,127.34,127.79,129.03,129.78,129.85,130.14,133.12$, 133.29, 133.98, 135.36, 136.18, 136.55, 137.83, 138.61, 143.63, 144.62, 145.77, 148.09, 151.75 ppm; LC-MS (IT-TOF): $\mathrm{m} / \mathrm{z}=632\left(\mathrm{M}^{+}\right), t_{\mathrm{R}}=20 \mathrm{~min}$.

4-Chloro-N-(4,5-dihydro-5-oxo-1-phenyl-1H-1,2,4-triazol3-yl)-5-methyl-2-[4-(trifluoromethyl)benzylthio]benzene-

sulfonamide ( $35, \mathrm{C}_{23} \mathrm{H}_{18} \mathrm{ClF}_{3} \mathrm{~N}_{4} \mathrm{O}_{3} \mathrm{~S}_{2}$ )

Starting from $0.529 \mathrm{~g} 20(1 \mathrm{mmol})$ in $1 \mathrm{~cm}^{3} \mathrm{THF}$, the reaction mixture was refluxed for $9 \mathrm{~h}$. The product was purified to give $0.29 \mathrm{~g}(52 \%)$ of 35. M.p.: $208-210{ }^{\circ} \mathrm{C}$; TLC: $\quad R_{\mathrm{f}}=0.40 \quad$ (benzene:EtOH $=2: 1$ ); $\quad$ IR $\quad(\mathrm{KBr})$ : $\bar{v}=3,253(\mathrm{NH}), 2,923\left(\mathrm{CH}_{3}, \mathrm{CH}_{2}\right), 1,701(\mathrm{CO}), 1,327$, $1,127\left(\mathrm{SO}_{2}\right) \mathrm{cm}^{-1} ;{ }^{1} \mathrm{H}$ NMR $(500 \mathrm{MHz}$, DMSO-d 6 ): $\delta=2.35\left(\mathrm{~s}, 3 \mathrm{H}, \mathrm{CH}_{3}\right), 4.46\left(\mathrm{~s}, 2 \mathrm{H}, \mathrm{S}-\mathrm{CH}_{2}\right), 7.14(\mathrm{t}, 1 \mathrm{H}$, Ar), 7.36 (t, 2H, Ar), 7.50 (d, 2H, Ar), 7.56 (d, 2H, Ar), 7.59 (s, 1H, H-3), 7.63 (d, 2H, Ar), 7.97 (s, 1H, H-6), 11.99 $(\mathrm{s}, \quad 1 \mathrm{H}, \quad \mathrm{NH}) \mathrm{ppm} ;{ }^{13} \mathrm{C}$ NMR $\left(50 \mathrm{MHz}, \mathrm{DMSO}-d_{6}\right)$ : $\delta=19.21, \quad 35.92, \quad 117.62, \quad 124.70, \quad 125.36, \quad 125.43$, $127.76,129.15,129.83,130.11,133.17,133.25,135.78$, $135.97,137.88,138.89,139.63,144.53,151.51 \mathrm{ppm}$; LC-MS (IT-TOF): $m / z=554\left(\mathrm{M}^{+}\right), t_{\mathrm{R}}=17 \mathrm{~min}$.

4-Chloro-N-(4,5-dihydro-5-oxo-1-phenyl-1H-1,2,4-triazol3-yl)-5-methyl-2-(naphthalen-1-ylmethylthio)benzenesul-

fonamide (36, $\mathrm{C}_{26} \mathrm{H}_{21} \mathrm{ClN}_{4} \mathrm{O}_{3} \mathrm{~S}_{2}$ )

Starting from $0.513 \mathrm{~g} 21(1 \mathrm{mmol})$ in $1 \mathrm{~cm}^{3} \mathrm{THF}$, the reaction mixture was refluxed for $9 \mathrm{~h}$. After cooling to room temperature, the reaction mixture was treated with petroleum ether to obtain a white solid. The crude product was purified to give $0.166 \mathrm{~g}(31 \%)$ of 36. M.p.: $214-216{ }^{\circ} \mathrm{C}$; TLC: $R_{\mathrm{f}}=0.55\left(\mathrm{CHCl}_{3}: \mathrm{MeOH}=16: 3\right) ; \mathrm{IR}(\mathrm{KBr}): \bar{v}=3,258$ $(\mathrm{NH}), 2,922\left(\mathrm{CH}_{3}, \mathrm{CH}_{2}\right), 1,720(\mathrm{CO}), 1,349,1,168$
$\left(\mathrm{SO}_{2}\right) \mathrm{cm}^{-1} ;{ }^{1} \mathrm{H}$ NMR $\left(500 \mathrm{MHz}, \mathrm{DMSO}-d_{6}\right): \delta=2.39$ (s, $\left.3 \mathrm{H}, \mathrm{CH}_{3}\right), 4.80\left(\mathrm{~s}, 2 \mathrm{H}, \mathrm{S}-\mathrm{CH}_{2}\right), 7.15(\mathrm{t}, 1 \mathrm{H}, \mathrm{Ar}), 7.32-7.42$ (m, 3H, Ar), 7.51-7.53 (m, 3H, Ar), 7.62-7.73 (m, 3H, H-3, Ar), $7.83(\mathrm{~d}, 1 \mathrm{H}, \mathrm{Ar}), 7.92(\mathrm{~d}, 1 \mathrm{H}, \mathrm{Ar}), 8.00(\mathrm{~s}, 1 \mathrm{H}, \mathrm{H}-6), 8.20$ (d, 1H, Ar), $11.90(\mathrm{~s}, 1 \mathrm{H}, \mathrm{NH}) \mathrm{ppm} ;{ }^{13} \mathrm{C}$ NMR $(50 \mathrm{MHz}$, DMSO- $\left.d_{6}\right)$ : $\delta=19.26,35.02,117.70,124.41,124.78$, $125.69,126.25,126.62,128.44,128.58,128.78,129.21$, $129.56,131.43,131.61,132.95,133.11,133.64,135.66$, $137.08,137.91,138.95,139.51,151.50 \mathrm{ppm} ;$ LC-MS (IT-TOF): $\mathrm{m} / \mathrm{z}=536\left(\mathrm{M}^{+}\right), t_{\mathrm{R}}=18 \mathrm{~min}$.

4-Chloro-N-[1-(4-chlorophenyl)-4,5-dihydro-5-oxo- $1 \mathrm{H}$ 1,2,4-triazol-3-yl]-5-methyl-2-(naphthalen-1-ylmethylthio)benzenesulfonamide $\left(37, \mathrm{C}_{26} \mathrm{H}_{20} \mathrm{Cl}_{2} \mathrm{~N}_{4} \mathrm{O}_{3} \mathrm{~S}_{2}\right)$

Starting from $0.545 \mathrm{~g} 22(1 \mathrm{mmol})$ in $2 \mathrm{~cm}^{3} \mathrm{THF}$, the reaction mixture was refluxed for $9 \mathrm{~h}$. The product was purified to give $0.224 \mathrm{~g}(39 \%)$ of 37. M.p.: $205-206{ }^{\circ} \mathrm{C}$; TLC: $R_{\mathrm{f}}=0.59$ (benzene:EtOH $=2: 1$ ); IR $(\mathrm{KBr}): \bar{v}=3,251(\mathrm{NH}), 2,924$ $\left(\mathrm{CH}_{3}, \mathrm{CH}_{2}\right), 1,722(\mathrm{C}=\mathrm{O}), 1,352,1,166\left(\mathrm{SO}_{2}\right) \mathrm{cm}^{-1} ;{ }^{1} \mathrm{HNMR}$ $\left(200 \mathrm{MHz}, \mathrm{DMSO}-d_{6}\right): \delta=2.39\left(\mathrm{~s}, 3 \mathrm{H}, \mathrm{CH}_{3}\right), 4.81(\mathrm{~s}, 2 \mathrm{H}$, S- $\left.\mathrm{CH}_{2}\right), 7.31-7.58(\mathrm{~m}, 6 \mathrm{H}, \mathrm{Ar}), 7.62-7.72(\mathrm{~m}, 3 \mathrm{H}, \mathrm{Ar})$, 7.80-7.94 (m, 2H, Ar), 8.01 (s, 1H, H-6), 8.20 (d, 1H, Ar), 11.99 (s, $1 \mathrm{H}, \mathrm{NH}) \mathrm{ppm} ;{ }^{13} \mathrm{C}$ NMR $\left(50 \mathrm{MHz}\right.$, DMSO- $\left.d_{6}\right)$ : $\delta=19.27,34.99,119.09,124.41,125.89,126.24,126.61$, $128.41,128.56,128.77,129.17,129.56,131.43,131.60$, 132.96, 133.09, 133.64, 135.59, 136.76, 137.08, 138.98, 139.92, 142.10, $151.42 \mathrm{ppm}$; LC-MS (IT-TOF): $\mathrm{m} / \mathrm{z}=570$ $\left(\mathrm{M}^{+}\right), t_{\mathrm{R}}=22 \mathrm{~min}$.

4-Chloro-N-[4,5-dihydro-1-(4-methylphenylsulfonyl)-5oxo-1H-1,2,4-triazol-3-yl]-5-methyl-2-(naphthalen-1ylmethylthio)benzenesulfonamide $\left(\mathbf{3 8}, \mathrm{C}_{27} \mathrm{H}_{23} \mathrm{ClN}_{4} \mathrm{O}_{5} \mathrm{~S}_{3}\right)$ Starting from $0.589 \mathrm{~g} 23(1 \mathrm{mmol})$ in $2 \mathrm{~cm}^{3}$ THF, the reaction mixture was refluxed for $11 \mathrm{~h}$. The product was purified to give $0.438 \mathrm{~g}$ (70 \%) of 38. M.p.: $118-120{ }^{\circ} \mathrm{C}$; TLC: $\quad R_{\mathrm{f}}=0.60 \quad$ (benzene:EtOH $\left.=2: 1\right) ; \quad$ IR $\quad(\mathrm{KBr})$ : $\bar{v}=3,530(\mathrm{NH}), 2,973\left(\mathrm{CH}_{3}, \mathrm{CH}_{2}\right), 1,726(\mathrm{CO}), 1,388$, $1,370,1,193,1,177\left(\mathrm{SO}_{2}\right) \mathrm{cm}^{-1} ;{ }^{1} \mathrm{H}$ NMR $(500 \mathrm{MHz}$, DMSO- $\left.d_{6}\right): \delta=2.31\left(\mathrm{~s}, 3 \mathrm{H}, \mathrm{CH}_{3}\right), 2.37\left(\mathrm{~s}, 3 \mathrm{H}, \mathrm{CH}_{3}\right), 4.70$ $\left(\mathrm{s}, 2 \mathrm{H}, \mathrm{S}-\mathrm{CH}_{2}\right), 7.32(\mathrm{~d}, 2 \mathrm{H}, \mathrm{Ar}), 7.38(\mathrm{t}, 1 \mathrm{H}, \mathrm{Ar})$, 7.48-7.53 (m, 5H, Ar), 7.63 (s, 1H, H-3), 7.84 (d, 1H, Ar), 7.90 (s, 1H, H-6), 7.94 (d, 1H, Ar), 8.08 (d, 1H, Ar), 11.90 (brs, $1 \mathrm{H}, \mathrm{NH}) \mathrm{ppm} ;{ }^{13} \mathrm{C}$ NMR $\left(50 \mathrm{MHz}\right.$, DMSO- $\left.d_{6}\right)$ : $\delta=19.29,21.42,35.06,124.28,125.76,126.27,126.64$, $127.37,128.27,128.56,128.79,128.96,130.19,131.38$, $131.59,132.90,133.27,133.64,133.91,135.68,136.75$, 138.77, 143.56, 145.85, 151.78 ppm; LC-MS (IT-TOF): $\mathrm{m} / \mathrm{z}=614\left(\mathrm{M}^{+}\right), t_{\mathrm{R}}=23 \mathrm{~min}$.

4-Chloro-N-(4,5-dihydro-5-oxo-1-phenyl-1H-1,2,4-triazol3-yl)-2-(1,2-dihydro-2-oxoquinolin-4-ylmethylthio)-5methylbenzenesulfonamide $\left(39, \mathrm{C}_{25} \mathrm{H}_{20} \mathrm{ClN}_{5} \mathrm{O}_{4} \mathrm{~S}_{2}\right)$ Starting from $0.528 \mathrm{~g} 24(1 \mathrm{mmol})$ in $3 \mathrm{~cm}^{3}$ THF, the reaction mixture was refluxed for $9 \mathrm{~h}$. The product was purified to give 
$0.161 \mathrm{~g}(29 \%)$ of 39. M.p.: $185-188{ }^{\circ} \mathrm{C}$; TLC: $R_{\mathrm{f}}=0.12$ $\left(\mathrm{CHCl}_{3}: \mathrm{MeOH}=16: 3\right), R_{\mathrm{f}}=0.19\left(\mathrm{CHCl}_{3}: \mathrm{MeCN}: \mathrm{AcOH}\right.$ $=2: 1: 0.05)$; IR (KBr): $\bar{v}=3,467(\mathrm{NH}), 2,923\left(\mathrm{CH}_{3}, \mathrm{CH}_{2}\right)$, 1,692, 1,655 (CO), 1,383, $1,127\left(\mathrm{SO}_{2}\right) \mathrm{cm}^{-1} ;{ }^{1} \mathrm{H} \mathrm{NMR}$ (500 MHz, DMSO- $\left.d_{6}\right): \delta=2.37\left(\mathrm{~s}, 3 \mathrm{H}, \mathrm{CH}_{3}\right), 4.59(\mathrm{~s}, 2 \mathrm{H}$, $\mathrm{S}-\mathrm{CH}_{2}$ ), $6.58(\mathrm{~s}, 1 \mathrm{H}, \mathrm{Ar}), 7.10(\mathrm{t}, 1 \mathrm{H}, \mathrm{Ar}), 7.16(\mathrm{t}, 1 \mathrm{H}, \mathrm{Ar}), 7.30$ (d, 1H, Ar), 7.34 (t, 2H, Ar), 7.49 (t, 1H, Ar), 7.55 (s, 1H, H-3), 7.65 (d, 2H, Ar), 7.88 (d, 1H, Ar), 8.01 (s, 1H, H-6), 11.73 (s, $2 \mathrm{H}, \mathrm{NH}$-quinolin, NH-triazolone) ppm; ${ }^{13} \mathrm{C}$ NMR $(50 \mathrm{MHz}$, DMSO- $\left.d_{6}\right): \delta=19.27,33.74,115.88,117.75,118.29$, $121.90,122.48,124.75,125.28,129.17,129.46,130.83$, $133.17,133.53,135.51,136.33,137.84,138.90,139.23$, 139.47, 145.59, 151.51, 161.49 ppm; LC-MS (IT-TOF): $m / z=554\left(\mathrm{M}^{+}\right), t_{\mathrm{R}}=17 \mathrm{~min}$.

4-Chloro-2-(2,3-dihydrobenzo[b][1,4]dioxin-2-ylmethylthio)-N-(4,5-dihydro-5-oxo-1-phenyl-1H-1,2,4-triazol-3$y$ l)-5-methylbenzenesulfonamide $\left(\mathbf{4 0}, \mathrm{C}_{24} \mathrm{H}_{21} \mathrm{ClN}_{4} \mathrm{O}_{5} \mathrm{~S}_{2}\right)$ Starting from $0.519 \mathrm{~g} 25$ (1 mmol) in $3 \mathrm{~cm}^{3} \mathrm{THF}$, the reaction mixture was refluxed for $9 \mathrm{~h}$. The product was purified to give $0.262 \mathrm{~g}(48 \%)$ of 40. M.p.: $185-188{ }^{\circ} \mathrm{C}$; TLC: $\quad R_{\mathrm{f}}=0.53 \quad$ (benzene:ethanol $\left.=2: 1\right)$; IR $\quad(\mathrm{KBr})$ : $\bar{v}=3,311(\mathrm{NH}), 2,922\left(\mathrm{CH}_{3}, \mathrm{CH}_{2}\right), 1,697(\mathrm{CO}), 1,334$, $1,165\left(\mathrm{SO}_{2}\right) \mathrm{cm}^{-1} ;{ }^{1} \mathrm{H}$ NMR $\left(500 \mathrm{MHz}, \mathrm{DMSO}-d_{6}\right)$ : $\delta=2.38\left(\mathrm{~s}, 3 \mathrm{H}, \mathrm{CH}_{3}\right), 3.36\left(\mathrm{dd}, 1 \mathrm{H}, \mathrm{S}-\mathrm{CH}_{2}\right), 3.46(\mathrm{dd}$, $\left.1 \mathrm{H}, \mathrm{S}-\mathrm{CH}_{2}\right), 4.00\left(\mathrm{dd}, 1 \mathrm{H}, \mathrm{O}-\mathrm{CH}_{2}\right), 4.26\left(\mathrm{dd}, 1 \mathrm{H}, \mathrm{O}-\mathrm{CH}_{2}\right)$, 4.31-4.35 (m, 1H, O-CH), 6.70-6.81 (m, 4H, Ar), 7.12 (t, 1H, Ar), 7.34 (t, 2H, Ar), 7.64 (d, 2H, Ar), 7.76 (s, 1H, $\mathrm{H}-3), 8.01$ (s, 1H, H-6), 12.01 (s, 1H, NH) ppm; ${ }^{13} \mathrm{C}$ NMR $\left(50 \mathrm{MHz}, \mathrm{DMSO}-d_{6}\right): \delta=19.29,33.64,66.26,72.02$, $117.15,117.29,117.69,121.61,121.70,124.74,129.14$, $129.83,133.09,133.61,135.83,136.62,137.83,139.12$, 139.50, 142.61, 142.99, 151.54 ppm; LC-MS (IT-TOF): $m / z=545\left(\mathrm{M}^{+}\right), t_{\mathrm{R}}=21 \mathrm{~min}$.

\section{$X$-ray structure determination}

Experimental diffraction data were collected on a KM4 CCD kappa-geometry diffractometer (Oxford diffraction), equipped with a Sapphire2 CCD detector. An enhanced $\mathrm{X}$-ray Mo $\mathrm{K} \alpha$ radiation source with a graphite monochromator was used. Determination of the unit cell and diffraction data collection were carried out at $120 \mathrm{~K}$ in a stream of dry nitrogen (Oxford CryoSystems). All calculations (data reduction, structure solution, and refinement) were carried out using CrysAlisPro [49] package. The structure was solved by direct methods, and all nonhydrogen atoms were refined with anisotropic thermal parameters by full-matrix least squares procedure based on $F^{2}$. Final refinements were carried out using the SHELX-97 package [50], run under control of WinGX program [51].
All hydrogen atoms were refined using isotropic model with $U_{\text {iso }}(H)$ values fixed to be 1.2 times $U_{\text {eq }}$ of $\mathrm{C}$ atoms for $\mathrm{CH}$ and $\mathrm{CH}_{2}$ and 1.5 times $U_{\text {eq }}$ for $\mathrm{CH}_{3}$. Bond lengths C-H were fixed at $0.98 \AA$ for methyl groups, and $0.95 \AA$ for methylene and methine groups; distances $\mathrm{N}-\mathrm{H}$ were set to $0.88 \AA$ A. Solvating water molecules generated an electron density peak of ca. 1.7 e $\AA^{-3}$. Because the electron density maximum is placed at a special position $(1 / 2, y, 1 / 4)$ localization of hydrogen atoms is additionally uncertain so we did not attempt to find $\mathrm{H}$ atoms. The occupation factor of oxygen atom $\mathrm{O} 10$ was refined freely to obtain 0.079 . One incorrect reflection $\left(\begin{array}{lll}-1 & 1 & 17\end{array}\right)$ was omitted.

Crystallographic data for the structure of 31Pyr reported in this article have been deposited with the Cambridge Crystallographic Data Center as supplementary publication no. CCDC868805. Copies of the data can be obtained free of charge on application to CCDC, 12 Union Road, Cambridge CB2 1EZ, UK [Fax: (+44) 1223-336-033; email: deposit@ccdc.cam.ac.uk].

Acknowledgments The authors are very grateful to Dr. Joel Morris, Ph.D., Chief Drug Synthesis \& Chemistry Branch, National Cancer Institute (Bethesda, MD), for the in vitro anticancer screening.

Open Access This article is distributed under the terms of the Creative Commons Attribution License which permits any use, distribution, and reproduction in any medium, provided the original author(s) and the source are credited.

\section{References}

1. Negwer M (1994) Organic-chemical drugs and their synonyms. Akademie Verlag, Berlin

2. Sławiński J, Bednarski P, Grünert R, Reszka P (2003) Polish J Chem 77:53

3. Sławiński J, Bednarski P, Reszka P (2004) Polish J Chem 78:369

4. Sławiński J (2004) Eur J Med Chem 39:179

5. Sławiński J, Gdaniec M (2005) Eur J Med Chem 40:377

6. Sławiński J, Brzozowski Z (2006) Eur J Med Chem 41:1180

7. Brzozowski Z, Sławiński J (2007) Polish J Chem 81:1419

8. Brzozowski Z, Sączewski F, Sławiński J (2007) Eur J Med Chem 42:1218

9. Brzozowski Z, Sączewski F, Sławiński J, Bednarski PJ, Grünert R, Gdaniec M (2007) Bioorg Med Chem 15:2560

10. Sławiński J, Brożewicz K, Fruziński A, Główka ML (2011) Heterocycles 83:1093

11. Brzozowski Z, Sławiński J, Kędzia A, Kwapisz E (2009) J Heterocycl Chem 46:1396

12. Sławiński J, Żołnowska B, Pirska D, Kędzia A, Kwapisz E (2011) J Enzyme Inhib Med Chem. doi:10.3109/14756366.2011.625024

13. Kuo CL, Assefa H, Brzozowski Z, Sławiński J, Sączewski F, Buolamwini IK, Neamati N (2004) J Med Chem 47:385

14. Brzozowski Z, Sławiński J, Sączewski F, Sanchez T, Neamati N (2008) Eur J Med Chem 43:1188

15. Sączewski F, Sławiński J, Kornicka A, Brzozowski Z, Pomarnacka E, Innocenti A, Scozzafava A, Supuran CT (2006) Bioorg Med Chem Lett 16:4846 
16. Sączewski F, Innocenti A, Brzozowski Z, Sławiński J, Pomarnacka E, Kornicka A, Scozzafava A, Supuran CT (2006) J Enzyme Inhib Med Chem 21:563

17. Sączewski F, Innocenti A, Sławiński J, Kornicka A, Brzozowski Z, Pomarnacka E, Scozzafava A, Supuran CT (2008) Bioorg Med Chem 16:3933

18. Casini A, Scozzafava A, Mastrolorenzo A, Supuran CT (2002) Curr Cancer Drug Targets 2:55

19. Supuran CT, Scozzafava A, Casini A (2003) Med Res Rev 23:146

20. Kivela AJ, Kivela J, Saarnio J, Parkkila J (2005) World J Gastroenterol 11:155

21. Supuran CT, Scozzafava A (2007) Bioorg Med Chem 15:4336

22. Supuran CT (2008) Nat Rev Drug Discov 7:168

23. Supuran CT, Casini A, Mastrolorenzo A, Scozzafava A (2004) Mini Rev Med Chem 4:625

24. Abbate F, Casini A, Owa T, Scozzafava A, Supuran CT (2004) Bioorg Med Chem Lett 14:217

25. Rostom SA (2006) Bioorg Med Chem 14:6475

26. Chen J, Liu T, Wu R, Lou J, Cao J, Dong X, Yang B, He Q, Hu Y (2010) Bioorg Med Chem 18:8478

27. Mullican MD, Wilson MW, Connor DT, Kostlan CR, Schrier DJ, Dyer RD (1993) J Med Chem 36:1090

28. Elipe MV, Huskey SE, Zhu B (2003) J Pharm Biomed Anal 30:1431

29. Sheppeck JE, Gilmore JL, Tebben A, Xue CB, Liu RQ, Decicco CP, Duan JJ (2007) Bioorg Med Chem Lett 17:2769

30. Oza V, Ashwell S, Brassil P, Breed J, Ezhuthachan J, Deng C, Grondine M, Horn C, Liu D, Lyne P, Newcombe N, Pass M, Read J, Su M, Toader D, Yu D, Yu Y, Zabludoff S (2012) Bioorg Med Chem Lett 22:2330

31. Demirbaş N, Ugurluoglu R, Demirbaş A (2002) Bioorg Med Chem 10:3717

32. Pomarnacka E, Koźlarska-Kędra I (2003) Il Farmaco 58:423

33. Adams ND, Aquino CJ, Chaudhari AM, Ghergurovich JM, Kiesow TJ, Parrish CA, Reif AJ, Wiggall K (2011) Triazolones as fatty acid synthase inhibitors. WO patent 2011103546, 25 Aug 2011

34. Adams ND, Aquino CJ, Chaudhari AM, Ghergurovich JM, Kiesow TJ, Parrish CA, Reif AJ, Wiggall K (2011) Chem Abstr 155:380343

35. Ying W, Du Z, Sun L, Foley KP, Proia DA, Blackman RK, Zhou D, Inoue T, Tatsuta N, Sang J, Ye S, Acquaviva J, Ogawa LS, Wada Y, Barsoum J, Koya K (2012) Mol Cancer Ther 11:475

36. Sławiński J (2001) Pol J Chem 75:1309

37. Sheppeck JE, Gilmore JL, Tebben A, Xue CHB, Liu RQ, Decicco CP, Duan JJW (2007) Bioorg Med Chem Lett 17:2769

38. Shalini M, Yogeeswari P, Sriram D, Stables JP (2009) Biomed Pharmacother 63:187

39. Brown RJ, Annis G, Casalnuovo A, Chan D, Shapiro R, Marshall WJ (2004) Tetrahedron 60:4361

40. Brenner M, Bechtel WD, Palluk R, Wienrich M, Weiser NO (2002) Pharmaceutical compositions containing triazolones and methods of treating neurodegenerative disease using triazolones. US patent 6,492,407 B2, 10 Dec 2002

41. Huisgen R (1961) Proc Chem Soc 357

42. Galishev VA, Chistoklevtov UN, Petrov AA (1980) Russ Chem Res 49:880

43. Kuninobu Y, Nishimura S, Takai K (2006) Org Biomol Chem 4:203

44. Tsuge O, Kanemasa S, Tashiro M (1968) Tetrahedron 24:5205

45. Tsuge O, Kanemasa S (1972) Bull Chem Soc Jpn 45:2877

46. Macrae CF, Bruno IJ, Chisholm JA, Edgington PR, McCabe P, Pidcock E, Rodriguez-Monge L, Taylor R, van de Streek J, Wood PA (2008) J Appl Crystallogr 41:466

47. Boyd RM, Paull KD (1995) Drug Dev Res 34:91

48. Amos LA (2011) Semin Cell Dev Biol 22:916

49. Oxford Diffraction (2005) CrysAlis CCD and CrysAlis RED, Version 1.171. Oxford Diffraction Ltd, Abingdon, England

50. Sheldrick GM (2008) Acta Crystallogr A 64:112

51. Farrugia LJ (1999) J Appl Crystallogr 32:837 